
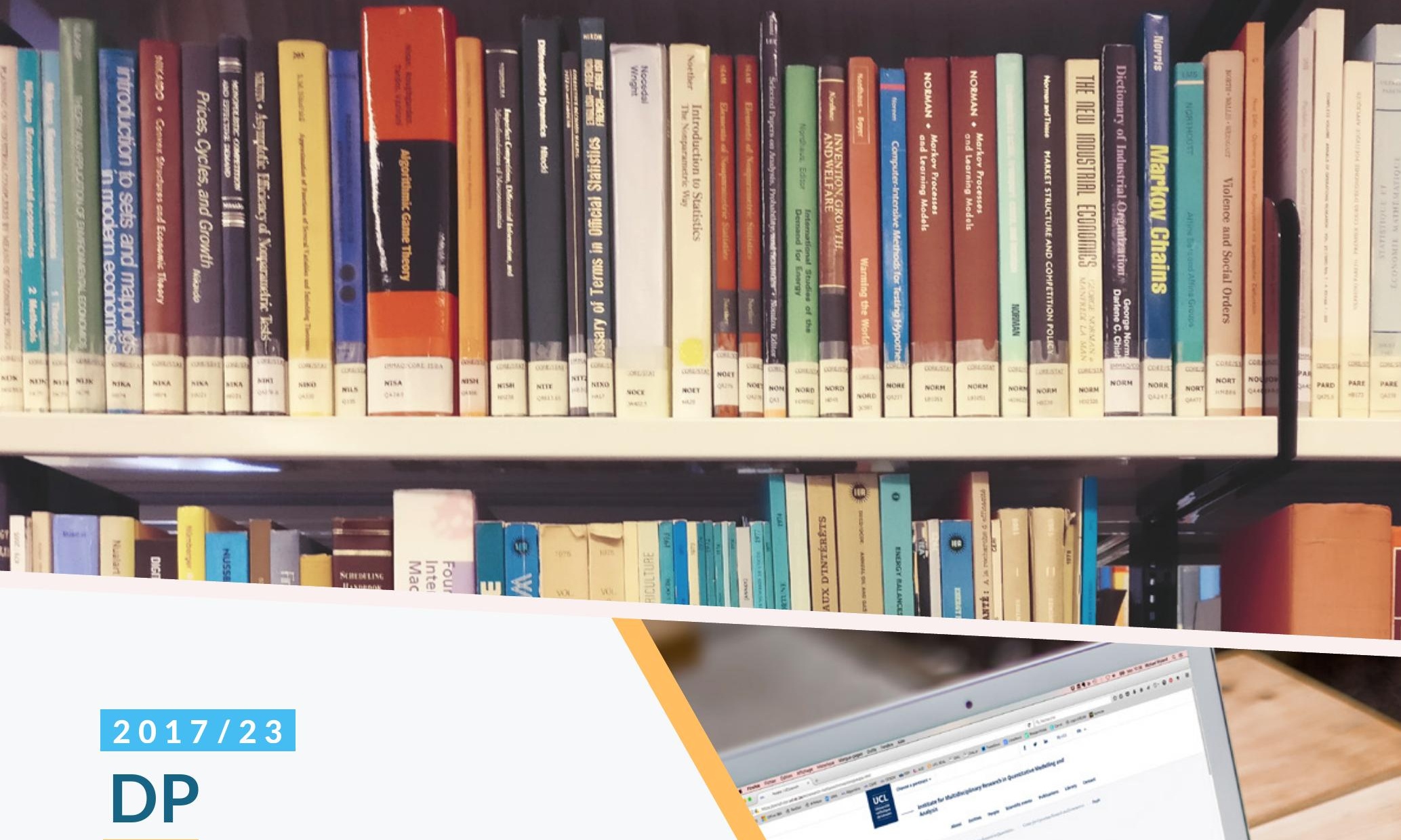

Alejandro Lamas and Philippe Chevalier

Joint dynamic pricing and lot-sizing under competition

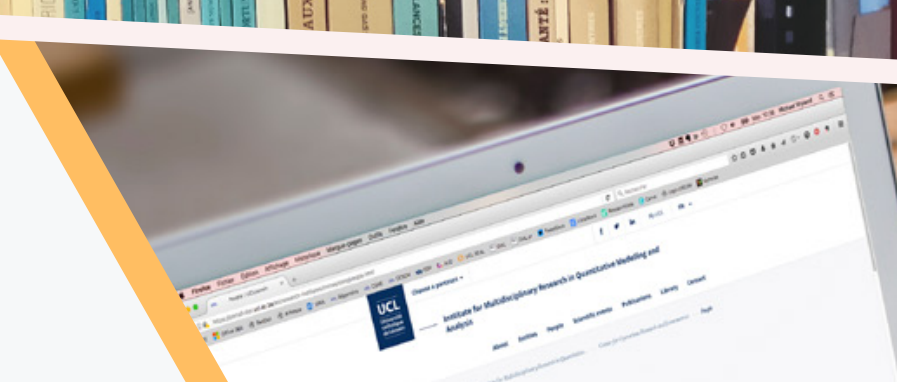




\section{CORE}

Voie du Roman Pays 34, L1.03.01

Tel (32 10) 474304

Fax (32 10) 474301

Email: immaq-library@uclouvain.be

https://uclouvain.be/en/research-institutes/

immaq/core/discussion-papers.html 


\title{
Joint Dynamic Pricing and Lot-Sizing under Competition
}

\author{
Alejandro Lamas ${ }^{\mathrm{a}, *}$, Philippe Chevalier ${ }^{\mathrm{b}}$, \\ ${ }^{a}$ NEOMA Business School, 1 Rue du Maréchal Juin, 76825 Mont-Saint-Aigean Cedex, France \\ ${ }^{b}$ Louvain School of Management - Center for Operations Research and Econometrics, Université catholique de Louvain, \\ Place des Doyens 1, 1348 Louvain-la-Neuve, Belgium
}

\begin{abstract}
We study the joint dynamic pricing and lot-sizing problem when firms operate in a competitive environment. Bearing in mind that a dynamic pricing strategy is successful when customers understand it, we assume each firm selects prices from a discrete set. The problem corresponds to a Bertrand model, so the pricing strategies of the firms should constitute a Nash equilibrium. Given the combinatorial nature of the decisions, computing the equilibrium in a tractable time may not be feasible for larger instances. In order to compute the equilibrium efficiently, we propose a framework consisting of solving iteratively Mixed Integer Programming formulations. The framework reduces the complexity of the problem by using the fact that pricing and inventory planning remain stable to marginal variations in competitors' prices.

Keywords: Production, dynamic pricing, competition, lot-sizing, joint production/marketing decisions
\end{abstract}

\section{Introduction}

Setting the right price for a product is one of the keys to a successful business. Although microeconomics began proposing pricing models long ago, pricing has become even more challenging because the proliferation of products that can often be substituted for one another. Thus, firms seeking to sustain a competitive position should implement aggressive price strategies to maximize their revenues while meeting the demand efficiently. One such price strategy increasingly adopted is that of dynamic pricing, by which the posted price of a product varies over time and provides the firm with dual benefits: it extracts the maximum surplus from customers and influences the demand to control its operating costs. A dynamic pricing strategy cannot be made in isolation, because the efficiency of such a strategy depends on how customers and competitors react to the prices (see Elmaghraby and Keskinocak (2003) and Yano and Gilbert (2005)). Hence, a firm has to consider the interaction with its competitors when pricing its products.

\footnotetext{
${ }^{*}$ Corresponding author. Tel.:+33 232825869

Email addresses: alejandro.lamas@neoma-bs.fr (Alejandro Lamas), philippe.chevalier@uclouvain.be (Philippe Chevalier)
} 
Moreover, pricing cannot be made in isolation from the firm's other activities. For instance, pricing depends on production/inventory planning, because this planning will determine the cost structure that will be used as an input for price setting. The inverse relationship also holds; production/inventory planning should meet the demand resulting from the firm's pricing strategy. Therefore, decision-making processes should plan pricing and production/inventory activities simultaneously. This is typically the goal of the Sales \& Operations Planning (S\&OP) process that is increasingly adopted by companies. Some hurdles, however, arise from the computational resources to implement a simultaneous optimization approach. In general, production/inventory planning is characterized by economies of scale and may involve discrete decisions, such as when and how much to order, so the time required for optimal planning can increase with the length of the planning horizon.

Another two elements characterize simultaneous dynamic pricing and inventory/manufacturing planning. First, in actual business environments the success of dynamic pricing depends on how the consumer understands and manages pricing signals (Bonsall et al., 2007); so a reduced set of prices helps pricing transparency, customer satisfaction and long-term loyalty (Mauri, 2007). Two practical illustrations of discrete dynamic pricing are provided by the fashion business, with discounts of $70 \%, 50 \%$ or $20 \%$; and the power supply industry, with low-demand, medium-demand and high-demand prices (Lanquepin-Chesnais et al., 2012). Thus, it is natural to model prices as discrete decision variables.

Second, the competitive environment is another factor influencing the complexity of simultaneous pricing and inventory/manufacturing planning. As each competitor aims to maximize its profit and there is a mutual substitution effect between the products on offer, the competitive environment corresponds to a Bertrand competition, i.e. competitors will assume that others will not change their prices in response to its price cuts. Thus, the pricing strategies posted by competitors should constitute a Nash Equilibrium (NE) (Nash, 1950). Reaching an NE will increase the complexity of the problem, because it requires computing the payoff matrices of the firms for any combination of potential pricing strategies and these computations increase with the set of available prices and the length of the horizon.

We propose a tractable time approach for simultaneous dynamic pricing and production/inventory planning in competitive environments. We represent the substitution effect between competitors by assuming that the demand for each depends on the prices posted by each competitor. We model each competitor's production/inventory activities as a dynamic lot-sizing problem (LSP), i.e., we determine when and how much to produce in order to minimize ordering, production and holding costs over a finite horizon consisting of discrete periods (see Wolsey (1995); Karimi et al. (2003); Brahimi et al. (2006) for 
an extended literature review of this problem). For any pricing strategy of the competitor, an LSP should be solved to construct the full payoff matrices. These computations can increase the time required to obtain an NE, so we set aside unnecessary calculations by extending the LSP formulation to obtain the best pricing and production/inventory response for each pricing strategy of the competitors . In addition, given that the best pricing and production/inventory response does not vary significantly with marginal variations in competitors' pricing strategies, we can discard pricing strategies that will not constitute an NE before computations are made. We, therefore, reach an NE by partially calculating the payoff matrices of the competitors. Our numerical experiments provide evidence of the significant time savings that can be achieved by implementing the proposed selective revision of the payoff matrices.

Our paper contributes to the scientific discussion by introducing joint dynamic pricing and lot-sizing under competition and a discrete set of prices. We also provide a novel approach for determining pricing strategies that combines pricing decisions, discrete mathematical programming and game theory. In particular, we characterize the payoff matrix of the firms by combining a Mixed Integer Programming (MIP) formulation for identifying dominated pricing strategies and the information of already revised pricing strategies. The characterization helps to reduce computational time significantly compared with a full revision of the payoff matrices. Our paper also helps practitioners, because the proposed framework enables them to solve larger instances of the problem in tractable time. Moreover, this paper builds bridges between theoretical micro-economic models and actual business operations; in particular, we adapt the classical pricing/cost equilibrium to settings in which firms operate in the presence of economies of scale and need to make discrete decisions. Further, our numerical experiments provide evidence of the advantages of dynamic pricing and modeling competition.

\section{Literature Review}

The literature on both operations management and marketing pays particular attention to the need for stronger coordination between production and marketing decisions. Upasani and Uzsoy (2007) classify existing research on the production/marketing interface into three main streams: (1) joint price-production quantity determination, (2) promotion planning and coordination, and (3) price, capacity and lead time models. We focus on the first research stream, where firms subject their price and production planning decisions to both operations and marketing constraints; the significance of the stream lies on the fact that an approach that cordinate such decisions is not only usefu, but essential for a firm (Chan et al., 2004). Since a firm's production planning implies management of the stock of finished products, we use 
the concept of joint pricing and production/inventory planning.

We start by classifying the existing research on joint price-production quantity according to their time modeling: continuous time models and discrete time models.

The first group of approaches to simultaneously planning pricing and production/inventory assumes continuous time horizons. The early work of Whitin (1955) constitutes the first quantitative approach to such planning. Within assumes price sensitive demand and uses the well-known Economic Order Quantity (EOQ) problem to model production/inventory operations. The goal is to set a single unit price $p$ and a re-order quantity $Q$ in order to maximize profits. Cheng (1990) and Chen and Min (1994) extend Within's model to multi-item settings. The authors provide the Kuhn-Tucker optimality conditions when both storage space and inventory investment are limited. Lee (1994) includes inventory investment budget and shows that computational time grows almost linearly with problem size. Kim and Lee (1998); Lee (1993) and Jung and Klein (2006) propose Geometric Programming approaches for the single item joint EOQ and pricing problem.

In the same stream of continuous time horizons, Pekelman (1974) addresses simultaneous dynamic pricing and time-dependent production quantities. Here, production rate $q(t)$ and optimal price $p(t)$ at time $t$ are the decision variables. Vanthienen (1975) and Feichtinger and Hartl (1985) extend Pekelman's model by including capacity constraints and by allowing backlog, respectively. Chen and Chu (2003) adapt pricing and production/inventory planning to frequent market updates. Their results are interesting for the launch of new products, because sales rates are highly sensitive to price strategies. Ray et al. (2005) study the EOQ problem when demand corresponds to linear or log-linear functions of the prices; the authors state, counter-intuitively, that batch size is not necessarily monotone increasing in set-up cost. In order to deal with demand uncertainty, Adida and Perakis (2006) extend the nominal formulation by taking a robust optimization approach.

The second group of approaches assumes the horizon to be a set of discrete periods. Most of these approaches consider the LSP as the most suitable model for representing production/inventory planning. Thomas (1970) studies simultaneous dynamic pricing and LSP. Through an analytical characterization of optimal solutions, the author reduces the computations required to obtain the optimal pricing. In order to simplify the dynamic pricing decision, some approaches assume a single price for the whole horizon. For instance, Kunreuther and Schrage (1973); Gilbert (1999) study the joint single price and LSP decisions and Gilbert (2000) extends this problem for multiple items that share a common capacity. When prices can vary through time, Bhattacharjee and Ramesh (2000) propose heuristic approaches for 
the uncapacitated version of the problem. Martel and Gascon (1998) deal with the problem of maximizing profits when operations are uncapacitated and the unit inventory holding costs in a period is a function of the procurement decisions made in previous periods. The authors derive an $O\left(T^{2}\right)$ algorithm which provides a probably optimal solution of an integer linear programming formulation of the general problem. For capacitated operations, Deng and Yano (2006) characterize the optimal solution and propose an algorithm whose complexity is slightly greater than the traditional LSP. The authors also show that larger capacity may lead to higher optimal prices. Haugen et al. (2007) propose a Lagrangian relaxation procedure to solve the problem for capacitated operations. For the same problem, Gonzalez-Ramirez et al. (2011) propose a Dantzig-Wolfe decomposition where the capacity constraints are the linking constraints. Onal and Romeijn (2011) extend the capacitated problem to the multi-item case. They develop two alternative Dantzig-Wolfe decomposition formulations and propose to solve the relaxations using column generation and the overall problem using branch-and-price. The authors test such formulations for both dynamic and static pricing strategies. Similar to our assumptions (but considering the decisions of a firm in isolation), Lanquepin-Chesnais et al. (2012) study a single item problem where a firm maximizes its profit over a discrete set of prices. The authors propose a Lagrangian relaxation to solve this problem.

Although there is a vast literature on simultaneous pricing and production/inventory planning, the scientific discussion has paid less attention to this problem in competitive environments. Min (1992); Chen et al. (1995) were the first authors to investigate simultaneous pricing and production/inventory planning under competition. These authors assume that each competitor must solve an EOQ problem and that their products compete for the same buyers. Cachon and Harker (2002) study a duopoly where competitors operate in a situation where economies of scale are possible, such as in the case of the EOQ model. The authors derive general conditions for the existence of one equilibrium at most between competitors. Transchel and Minner (2011) propose an extension of Cachon and Harker (2002) by assuming that one firm has an EOQ cost structure, and another implements a just-in-time policy. Adida and Perakis (2010) address the problem where two firms compete through dynamic pricing and inventory control and where demand is uncertain. These authors introduce a demand base fluid model where the demand is a linear function of the price of the supplier and of her competitor, the inventory and production costs are quadratic, and all coefficients are time dependent.

More related to our work, several papers focuses on discrete time decisions in competitive environments. Fredergruen and Meissner (2009) study the competition between firms when their production lines can be modeled as a capacitated LSP. The authors prove the existence of price equilibrium and associated 
optimal dynamic lot-sizing plans, under mild conditions. In contrast to our work, they assume that each firm sets a single price for the whole production period. The constant price condition is relaxed in Pedroso and Smeers (2010). The authors propose a fixed-point iteration to achieve a duopoly equilibrium when competitors produce perfect substitute items. Our work differs from this in three ways: we assume that competitors select prices from a discrete set; we examine the possibility of randomizing price strategies (Mixed Nash Equilibrium); and we assume that competitors offer non-perfect substitutes products. To the best of our knowledge, our paper is the first attempt to include such dimensions in discrete time problems.

\section{Problem Description}

\subsection{Problem parameters}

Consider two self-interested firms, Firm 1 and Firm 2, that manufacture and sell interchangeable products, so the demand for these products depends on the prices set by each firm. The firms therefore maximize their profits by making pricing decisions that take into account both their operating costs and the competitor's price.

We assume that each firm plans its production for $N$ consecutive periods, which we call a planning block. The dynamics for a planning block are as follows. Note that, in our description Firm $i$ represents either Firm 1 or Firm 2, and Firm $j$ represents its competitor.

- Before carrying out the production for the planning block, each firm decides the unit selling price of its product for each period. Firm $i$ selects such prices from the discrete set $M_{i}$ consisting of $K_{i}$ elements, where $M_{i}=\left\{m_{i, 1}, m_{i, 2}, \ldots, m_{i, K_{i}}\right\}$. Without loss of generality, we assume $m_{i, k}<$ $m_{i, k+1}, \forall k=1, \ldots, K_{i}-1$. The pricing decision of Firm $i$ for the planning block is what we call a pure pricing strategy, and we represent it by $s_{i} \in \mathbf{R}^{N}$, such that $s_{i, n} \in M_{i}$. We denote the set of all pure pricing strategies of Firm $i$ by $S_{i}$. Note that $\left|S_{i}\right|=\left(K_{i}\right)^{N}$, so the number of available strategies grows exponentially with the planning block length.

- The pure pricing strategies selected by each firm induce their demand for each period in the planning block. We use $d_{i}: S_{i} \times S_{j} \rightarrow \mathbf{R}^{N}$ to represent the relationship between price and demand for Firm $i$, in which demand is a linear function of prices, as we show in equation (1).

$$
d_{i, n}\left(s_{i}, s_{j}\right)=\alpha_{i, n}-\beta_{i, n} \cdot s_{i, n}+\gamma_{i, n} \cdot s_{j, n}, \quad \forall i=1,2, j \neq i
$$


$\alpha_{i, n}$ represents the demand for Firm $i$ when the prices of both firms are equal to 0 in period $n$; $\beta_{i, n}$ is the sensitivity of demand for Firm $i$ to its product price in period $n$. We assume an inverse relation between price and firm demand. $\gamma_{i, n}$ is the sensitivity of demand for Firm $i$ to its competitor's price in period $n$. Since the firms offer interchangeable products, we establish that $\gamma_{i, n} \geq 0$.

- Once the firms have priced their products, they produce according to their demand for each period in the planning block. Each firm organizes its production to meet the demand while minimizing operating costs. We assume that the operating costs for each firm is derived from solving an $L S P$.

The parameters of the $L S P$ faced by each firm are (note that for simplicity, we assume that the costs do not vary over time):

- $c_{i}$ : unit production cost of firm $i$.

- $f_{i}$ : setup cost of the line managed by firm $i$.

- $h_{i}$ : unit holding cost of firm $i$.

- $d_{i, n}\left(s_{i}, s_{j}\right)$ : demand faced by firm $i$ in period $n$ (see equation (1)).

The decision variables of the $L S P$ are $x, y, z \in \mathbf{R}^{2 \times N}$, such that:

- $x_{i, n}$ : production volume for firm $i$ in period $n$.

- $y_{i, n}: 1$ if the line managed by firm $i$ is used in period $n, 0$ otherwise.

- $z_{i, n}$ : amount of inventory of the item held by firm $i$ at the end of period $n$.

The set of constraints ensuring that the operations of Firm $i$ satisfy the demand derived from pure pricing strategies $s_{i}$ and $s_{j}$ are as follows:

$$
\begin{array}{r}
z_{i, n}=z_{i, n-1}+x_{i, n}-d_{i, n}\left(s_{i}, s_{j}\right) \quad \forall n \in N \\
x_{i} \leq M \cdot y_{i}, \\
x_{i}, z_{i} \geq 0, y_{i} \in\{0,1\},
\end{array}
$$

where constraint (2) represents inventory conservation and demand satisfaction; constraint (3) states that production can occur in a period only if such production is activated; in this constraint $M$ represents a 
large number. Let $\mathscr{X}_{i}\left(s_{i}, s_{j}\right)$ be the set of all the decisions $x_{i}$ that satisfy the constraints $(2)$ - (4) when the firms implement pure pricing strategies $\left(s_{i}, s_{j}\right)$.

Example 1. To illustrate our model and the proposed resolution method we will follow a little example with the following parameters:

Firm $i$ and Firm $j$ are planning their pricing and operations for the next 4 periods. The cost parameters of the firms are: $f_{i}=f_{j}=3, h_{i}=h_{j}=1$ and $c_{i}=c_{j}=0$. The demand function of both firms is constant across the time and poses the same sensitivity parameters $\beta_{i, n}=\beta_{j, n}=1, \forall n$ and $\gamma_{i, n}=\gamma_{j, n}=0.5, \forall n$; the demand functions, however, differ between each other in terms of the free parameter: $\alpha_{i, n}=5, \forall n$ and $\alpha_{j, n}=4, \forall n$. For each period $n$ the firms have to choose a price from the sets: $M_{i}=\{3,4,5\}$ and $M_{i}=\{2,3,4\}$.

For instance, if the firms choose the strategies $s_{i}=\{3,3,3,3\}$ and $s_{j}=\{2,2,2,2\}$, then the demand for Firm $i$ is $\{3,3,3,3\}$ the revenues of Firm $i$ correspond to 36 and the cost derived from the LSP is 12; thus, $\pi_{i, s_{i}, s_{j}}=24$. For the same pair of strategies, Firm $j$ has revenues of 28 and the cost derived from the LSP is 12; thus, $\pi_{j, s_{i}, s_{j}}=16$.

\subsection{The Competitive Lot-Sizing Problem}

In order to determine the resulting pricing and production decisions of both firms, we assume each firm has perfect information about its competitor's set of prices. So, the firms will choose prices that constitute an NE. Nevertheless, an NE may not exist, because the set of prices is discrete. In such a case, we assume that firms randomize their pricing. Firm $i$ will select the pure pricing strategy $s_{i} \in S_{i}$ with a certain probability. The probabilities that firms assign to each pure pricing strategy constitute a Mixed Nash Equilibrium (MNE).

In the remaining part of this section we describe our model of the decisions related to each firm's operations and pricing, and we formulate NE and MNE as sets of linear MIP constraints.

We define the function $C_{i}\left(x_{i}\right)$ that gives the total operating cost of Firm $i$ associated with the operational decision $x_{i} \in \mathscr{X}_{i}\left(s_{i}, s_{j}\right)$, as we show in expression (5).

$$
C_{i}\left(x_{i}\right)=c_{i} \cdot x_{i}^{T}+f_{i} \cdot y_{i}^{T}+h_{i} \cdot z_{i}^{T}
$$

Each firm expects that its pricing will lead to maximum profits. In other words, the goal is to maximize the difference between income and production/inventory costs. Given that the firms choose the pure pricing strategies $\left(s_{i}, s_{j}\right) \in S_{i} \times S_{j}$, we represent their income, production costs and profits by functions 
$R: S_{i} \times S_{j} \rightarrow \mathbf{R}^{2}, C^{o}: S_{i} \times S_{j} \rightarrow \mathbf{R}^{2}$ and $B: S_{i} \times S_{j} \rightarrow \mathbf{R}^{2}$, respectively, where $R_{i}\left(s_{i}, s_{j}\right)=s_{i} \cdot d_{i}\left(s_{i}, s_{j}\right)^{T}$, $C_{i}^{o}\left(s_{i}, s_{j}\right)=\min _{x_{i}}\left\{C_{i}\left(x_{i}\right) \mid x_{i} \in \mathscr{X}_{i}\left(s_{i}, s_{j}\right)\right\}$ and $B_{i}\left(s_{i}, s_{j}\right)=R_{i}\left(s_{i}, s_{j}\right)-C_{i}^{o}\left(s_{i}, s_{j}\right)$.

Next, we describe the problems of selecting and randomizing pure pricing strategies.

\subsubsection{Pure pricing strategies}

Each firm takes into account its known competitor's pricing $s_{j} \in S_{j}$ when deciding its own price to maximize profits, i.e., $\max _{s_{i}}\left\{B_{i}\left(s_{i}, s_{j}\right) \mid\left(s_{i}, s_{j}\right) \in S_{i} \times S_{j}\right\}$. Since the firms' prices are mutually dependent, they should choose pure pricing strategies that satisfy the conditions for an NE. The pair $\left(s_{i}, s_{j}\right)$ is an NE if $B_{i}\left(s_{i}, s_{j}\right) \geq B_{i}\left(s_{i}^{\prime}, s_{j}\right), \forall s_{i}^{\prime} \in S_{i}$ and $\forall i=1,2 ; j \neq i$. In other words, a firm has no incentive to change its pure pricing strategy unless its competitor's strategy changes.

\subsubsection{Randomizing pure pricing strategies}

Here, each firm estimates the probability that its pure pricing strategy will maximize its profits. Let $p_{i} \in[0,1]^{\left|S_{i}\right|}$ be the vector containing the probability that Firm $i$ assigns to its pure pricing strategy. $\max _{p_{i}}\left\{\sum_{s_{i} \in S_{i}} p_{i, s_{i}} \cdot \sum_{s_{j} \in S_{j}} p_{j, s_{j}} \cdot B_{i}\left(s_{i}, s_{j}\right) \mid \sum_{s_{i} \in S_{i}} p_{i, s_{i}}=1 \wedge p_{i, s_{i}} \geq 0, \forall s_{i} \in S_{i}\right\}$ represents the pricing problem of Firm $i$ for given $p_{j}$. Note that the constraints of this problem ensure that $p_{i}$ is a valid probability distribution.

Since the probability assignments are mutually dependent, the solution of the pricing problem should satisfy the MNE condition. $\left(p_{i}, p_{j}\right)$ is an MNE if $\sum_{s_{i} \in S_{i}} p_{i, s_{i}} \cdot \sum_{s_{j} \in S_{j}} p_{j, s_{j}} \cdot B_{i}\left(s_{i}, s_{j}\right) \geq \sum_{s_{i} \in S_{i}} p_{i, s_{i}}^{\prime}$. $\sum_{s_{j} \in S_{j}} p_{j, s_{j}} \cdot B_{i}\left(s_{i}, s_{j}\right), \forall p_{i}^{\prime}$ and $\forall i=1,2 ; j \neq i$. In other words, a firm has no incentive to change its mixed strategy unless its competitor's mixed strategy changes. Nash (1950) proves that at least one equilibrium exists for this game.

\subsubsection{Nash Equilibrium and Mixed Nash Equilibrium}

Sandholm et al. (2005) propose constraints (6) - (11) to describe an MNE. Two facts support Sandholms' formulation: in any equilibrium, every pure strategy is either played with probability 0, or has 0 regret; and, any vector of mixed strategies for the players where every pure strategy is either played with probability 0, or has 0 regret, is an equilibrium. By regret of a strategy, we mean the difference between the payoff derived from such strategy and the optimal payoff. Sandholms' formulation uses two parameters: $\pi \in$ $\mathbf{R}^{2 \times\left|S_{i}\right| \times\left|S_{j}\right|}$ such that $\pi_{i, s_{i}, s_{j}}=B_{i}\left(s_{i}, s_{j}\right)$ and $\bar{\pi} \in \mathbf{R}^{2}$, such that $\bar{\pi}_{i}=\max _{s_{i}^{l}, s_{i}^{h} \in S_{i}, s_{j}^{l}, s_{j}^{h} \in S_{j}}\left(\pi_{i, s_{i}^{h}, s_{j}^{h}}-\pi_{i, s_{i}^{l}, s_{j}^{h}}\right)$. Although, the decision variables correspond to the vector of probabilities $p_{i}, \forall i=1,2$, the authors also use variables $b_{i}, r_{i} \in \mathbf{R}^{\left|S_{i}\right|}, u_{i} \in\{0,1\}^{\left|S_{i}\right|}$ and $\bar{b}_{i} \in \mathbf{R}$, where: 
- $b_{i, s_{i}}$ : expected profit of Firm $i$ when implementing strategy $s_{i} \in S_{i}$.

- $r_{i, s_{i}}$ : expected regret of Firm $i$ when playing strategy $s_{i} \in S_{i}$.

- $u_{i, s_{i}}: 1$ if Firm $i$ plays the strategy $s_{i} \in S_{i}$ with probability 0; 0 otherwise.

- $\bar{b}_{i}$ : highest possible expected profit that Firm $i$ can obtain given the competitor's strategies.

$$
\begin{gathered}
\sum_{s_{i} \in S_{i}} p_{i, s_{i}}=1, \quad \forall i=1,2, \\
b_{i}^{T}=\pi_{i} \cdot p_{j}^{T} \quad \forall i=1,2 ; j \neq i, \\
r_{i, s_{i}}=\bar{b}_{i}-b_{i, s_{i}} \quad \forall s_{i} \in S_{i} ; \forall i=1,2, \\
p_{i, s_{i}} \leq 1-u_{i, s_{i}} \quad s_{i} \in S_{i} ; \forall i=1,2, \\
r_{i, s_{i}} \leq \bar{\pi}_{i} \cdot u_{i, s_{i}} \quad \forall s_{i} \in S_{i} ; \forall i=1,2, \\
p_{i}, b_{i}, \bar{b}_{i}, r_{i} \geq 0 ; u_{i} \in\{0,1\} \quad \forall i=1,2 .
\end{gathered}
$$

Constraint (6) ensures that $p_{i}$ is a valid probability distribution. Constraints (7) - (8) define the regret of a strategy. Constraint (9) ensures that $u_{i, s}=1$ only if $p_{s, i}=0$. Constraint (10) states that the regret of a strategy equals 0 , unless the strategy is played with probability 0 . Even though this formulation characterizes an MNE, we can make it an NE by including the constraint $p_{i} \in\{0,1\}, \forall i=1,2$ in Sandholm's formulation.

Sandholm's formulation provides pricing strategies that represent specific equilibriums between firms. For instance, solving the MIP models resulting from $\max \left(\bar{b}_{i}+\bar{b}_{j}\right)$ subject to (6) - (11) will lead to the equilibrium that maximizes the firms' joint profits. If we set the objective function $\min \left(\bar{b}_{i}+\bar{b}_{j}\right)$, the equilibrium minimizes the firms' joint profit. We can use other objective functions, including $\min \left(\bar{b}_{i}\right)$ (minimizing the profit of a firm), $\max \left(\bar{b}_{i}\right)$ (maximizing the profit of a firm), and $\operatorname{maxmin}\left\{\bar{b}_{i}, \bar{b}_{j}\right\}($ balancing the profits of the firms).

When modeling joint pricing and production/inventory planning, Sandholm's formulation may become computationally intensive, because the values $\left|S_{1}\right|$ and $\left|S_{2}\right|$ increase exponentially with $N, K_{1}$ and $K_{2}$. Thus, the formulation consists of $3 \cdot\left(K_{1}^{N}+K_{2}^{N}\right)+2$ continuous variables and $K_{1}^{N}+K_{2}^{N}$ binary variables. Moreover, Sandholm's formulation requires the full payoff mapping $B$ as a parameter, which necessitates the solution of $2 \cdot\left(K_{1} \cdot K_{2}\right)^{N} L S P$ s. 
We propose a methodology that reduces the effort for computing an equilibrium between the firms' price strategies. The methodology calculates either a NE or a MNE by comparing only the firms' best responses. Further, with the aim of shrinking the computation of a best response, we identify and discard dominated strategies from the set of potential equilibriums, so that we reduce both the number of variables of Sandholm's formulation and the number of $L S P$ s that should be solved. In Section 4 we describe and analyze the problem of finding the best response to each competitor's strategy.

Example 2. We continue with the same example introduced earlier. In order to reach a NE (or a MNE) with Sandholm's formulation, we have to compute for each firm the payoff resulting from $3^{4} \times 3^{4}=6,561$ combinations of pure pricing strategies of Firm $i$ and Firm $j$,i.e., for any combination of pure pricing strategies $s_{i}$ and $s_{j}$, we then compute the difference between the revenues derived from those strategies and the optimal cost of the corresponding LSP.

\begin{tabular}{ccccc}
\hline & \multicolumn{2}{c}{ Firm $i$} & \multicolumn{2}{c}{ Firm $j$} \\
\hline & Max. Payoff & Min. Payoff & Max. Payoff & Min. Payoff \\
$s_{i}$ & $\{3,3,3,3\}$ & $\{5,5,5,5\}$ & $\{5,5,5,5\}$ & $\{3,3,3,3\}$ \\
$s_{j}$ & $\{4,4,4,4\}$ & $\{2,2,2,2\}$ & $\{4,4,3,4\}$ & $\{2,3,4,4\}$ \\
$B\left(s_{i}, s_{j}\right)$ & 36 & 12 & 30 & 14.5 \\
$\bar{\pi}$ & \multicolumn{2}{c}{24} & \multicolumn{2}{c}{15.5} \\
\hline
\end{tabular}

Table 1: The strategies defining the regret function

Table 1 summarizes the maximum and the minimum payoff that Firm $i$ and Firm $j$ can obtain when planning pricing and operations. The rows $s_{i}$ and $s_{j}$ represents the strategies that lead the corresponding payoff. As we can observe in the Table 1, the maximum payoff for Firm $i$ is 36 and the minimum is 12, so the maximum regret is bounded by the difference between both numbers; therefore, we set the value of parameter $\bar{\pi}_{i}$ to $36-12=24$. In the same way, we fix the value of this parameter for Firm $j$ to $\bar{\pi}_{j}=30-14.5=15.5$.

The pricing strategies of the $N E$ are $s_{i}=\{3,4,4,4\}$ and $s_{j}=\{3,3,4,3\}$, the demand derived from those pricing strategies are $d_{i}=\{3.5,2.5,3.0,2.5\}$ and $d_{j}=\{2.5,3.0,2.0,3.0\}$; the production planning of Firm $i$ consists of producing 6.0 units in period 1 and 5.5 units in period 3, i.e., $x_{i}=\{6.0,0.0,5.5,0.0\}$ and the production of firm $j$ is given by $x_{j}=\{2.5,5.5,0.0,2.0\}$. The corresponding profits are $B_{i}\left(s_{i}, s_{j}\right)=31.5$ and $B_{j}\left(s_{i}, s_{j}\right)=22.5$.

\section{Best Response Problem}

We start by defining the best response of a firm to its competitor's pure pricing strategy. 
Definition 1. $s_{i}^{*}\left(s_{j}\right) \in S_{i}$ is the best response of the firm $i$ to $s_{j} \in S_{j}$ when $B_{i}\left(s_{i}^{*}\left(s_{j}\right), s_{j}\right) \geq B_{i}\left(s_{i}, s_{j}\right), \forall s_{i} \in$ $S_{i}$

We formulate a MIP for obtaining $s_{i}^{*}\left(s_{j}\right)$. To do so, we introduce the decision variable $w_{i} \in\{0,1\}^{K_{i} \times N}$, where $w_{i, k, n}$ is 1 if the firm $i$ selects the price $m_{i, k} \in M_{i}$ for period $n ; 0$ otherwise. We can express any $s_{i} \in S_{i}$ as a linear combination of $w$, such that $s_{i, n}\left(w_{i}\right)=\sum_{k=1}^{K_{i}} m_{i, k} \cdot w_{i, k, n}$. Then, we obtain the best response to the competitor's pricing strategy from the formulation (12) - (14).

$$
\begin{array}{ll}
\max _{w_{i}} & R_{i}\left(s_{i}\left(w_{i}\right), s_{j}\right)-C_{i}^{o}\left(s_{i}\left(w_{i}\right), s_{j}\right) \\
\text { s.t. } & \sum_{k=1}^{K_{i}} w_{i, k, n}=1, \quad \forall n=1, \ldots, N, \\
& w_{i, k, n} \in\{0,1\} \quad \forall n=1, \ldots, N, \quad \forall k=1, \ldots, K_{i} .
\end{array}
$$

Expression (12) gives the firm's maximum profit. Note that, $C_{i}^{o}\left(s_{i}(w), s_{j}\right)$ is the cost obtained by Firm $i$ when solving the $L S P$ resulting from choosing strategies $s_{i}(w)$ and $s_{j}$. Constraints $(13)-(14)$ state that Firm $i$ must select one and only one price per period.

Formulation (12)- (14) can help to reduce the computations of pricing equilibria, because it maps the payoff of the relevant pricing strategies by solving $\left(K_{1}\right)^{N}+\left(K_{2}\right)^{N}$ best response problems. As we explained above, the full payoff computation, requires the solution of $2 \cdot\left(K_{1} \cdot K_{2}\right)^{N} L S P$ s. Moreover, even though model (12) - (14) contains $K_{i} \cdot N$ new binary variables for each $L S P$, solving the problem may require fewer computations than for the full payoff mapping, because the full mapping is an iterative procedure that ignores the structure of the problem. Nevertheless, model (12) - (14) can express such a structure by implementing simple, well-known algorithms, such as Branch \& Bound or Branch \& Cut.

In the following two sub-sections, we show how to reduce computations even more by eliminating pricing strategies that can be shown not to be the best response to the competitor's strategy.

First, by analyzing small variations on the pricing strategy selected by the firm, we identify the pricing strategies that can be discarded as the best response to the competitor's strategy. Given that the analysis is born of the variations of the prices of the own firm, we call this active pruning.

Second, starting from the best response strategy to a given strategy of the competitor, we show how we can use the optimality conditions of the best response strategy to discard strategies as a response to small variations of the competitor's strategy. Thus, we may avoid revising the full set of strategies if we 
know the best response to a small variation of the competitor's strategy. We call this reactive pruning, as it is obtained from analyzing the variations of the prices of the competitor. We start by providing some definitions to clarify our presentation.

Definition 2. $\lambda\left(s_{i}^{\prime}, s_{i}, s_{j}\right)$ is the difference between the minimum costs of the operations of Firm $i$ when choosing strategies $s_{i}$ and $s_{i}^{\prime}$ as responses to the pure pricing strategy $s_{j} \in S_{j}$, i.e. $\lambda\left(s_{i}^{\prime}, s_{i}, s_{j}\right)=C_{i}^{o}\left(s_{i}^{\prime}, s_{j}\right)-$ $C_{i}^{o}\left(s_{i}, s_{j}\right)$.

Definition 3. $\tau\left(s_{j}^{\prime}, s_{i}, s_{j}\right)$ is the difference between the minimum cost of the operations of Firm $i$ by selecting the pure pricing strategy $s_{i} \in S_{i}$ as a response to $s_{j}$ and $s_{j}^{\prime} \in S_{j}$, i.e. $\tau\left(s_{j}^{\prime}, s_{i}, s_{j}\right)=C_{i}^{o}\left(s_{i}, s_{j}^{\prime}\right)-$ $C_{i}^{o}\left(s_{i}, s_{j}\right)$.

Besides these definitions, we use $\phi\left(s_{j}, n\right)=\frac{\alpha_{i, n}+\gamma_{i, n} \cdot s_{j, n}}{\beta_{i, n}}$ and $\Delta\left(s_{i}^{\prime}, s_{i}\right)=\sum_{n=1}^{N}\left(s_{i, n}-s_{i, n}^{\prime}\right)$, where $\phi\left(s_{j}, n\right)$ is the maximum price that Firm $i$ can set in order to obtain non-negative demand in period $n$, given that the competitor chooses the pricing strategy $s_{j}$; and $\Delta\left(s_{i}^{\prime}, s_{i}\right)$ corresponds to the sum of the difference between the prices per period of the pure pricing strategies $s_{i}$ and $s_{i}^{\prime}$ (note that $\Delta\left(s_{i}^{\prime}, s_{i}\right)=s_{i, n^{\prime}}-s_{i, n^{\prime}}^{\prime}$ if $s_{i, n}=s_{i, n}^{\prime}, \forall n \neq n^{\prime} \wedge s_{i, n^{\prime}} \geq s_{i, n^{\prime}}^{\prime}$. We also assume that demand in a period cannot be negative for any combination of pure pricing strategies.

\subsection{Active Pruning}

Given that the competitor chooses the pure pricing strategy $s_{j} \in S_{j}$, we analyze the relative convenience for Firm $i$ of choosing between two pure pricing strategies. Firm $i$ will prefer the pure pricing strategy $s_{i}$ to $s_{i}^{\prime}$ when condition (15) holds, i.e. the additional revenues obtained through $s_{i}^{\prime}$ does not surpass the additional cost associated with such a strategy relative to $s_{i}$.

$$
R_{i}\left(s_{i}^{\prime}, s_{j}\right)-R_{i}\left(s_{i}, s_{j}\right)<\lambda_{i}\left(s_{i}^{\prime}, s_{i}, s_{j}\right)
$$

We cannot express equation (15) in a closed form, because it requires the solution of two $L S P$ s. Further, the complexity of solving an $L S P$ increases with $N$, so we would like to avoid dealing directly with an $L S P$. Hence, we obtain closed form bounds for $\lambda\left(s_{i}^{\prime}, s_{i}, s_{j}\right)$, and we derive sufficient conditions for expression (15) to be satisfied.

Lemma 1. Given the pure pricing strategies $s_{i}, s_{i}^{\prime} \in S_{i}$ such that $s_{i, n}=s_{i, n}^{\prime}, \forall n \neq n^{\prime} \wedge s_{i, n^{\prime}}^{\prime} \leq s_{i, n^{\prime}}$, then $\underline{\lambda}\left(s_{i}^{\prime}, s_{i}\right)$ and $\bar{\lambda}\left(s_{i}^{\prime}, s_{i}, s_{j}\right)$ constitute lower and upper bounds for $\lambda\left(s_{i}^{\prime}, s_{i}, s_{j}\right)$, respectively, where 


$$
\underline{\lambda}\left(s_{i}^{\prime}, s_{i}\right)=c_{i} \cdot \beta_{i, n^{\prime}} \cdot \Delta\left(s_{i}^{\prime}, s_{i}\right),
$$

and

$$
\bar{\lambda}\left(s_{i}^{\prime}, s_{i}, s_{j}\right)=\underline{\lambda}\left(s_{i}^{\prime}, s_{i}\right)+\min \left\{h_{i} \cdot\left(n^{\prime}-1\right) \cdot \beta_{i, n^{\prime}} \cdot \Delta\left(s_{i}^{\prime}, s_{i}\right) \text { if } \exists n \in\left[1, n_{i}\right] \mid \phi\left(s_{j}, n\right)>m_{K_{i}}, f_{i}\right\}
$$

Proof. Given that $d_{i, n}\left(s_{i}^{\prime}, s_{j}\right)=d_{i, n}\left(s_{i}, s_{j}\right), \forall n \neq n^{\prime}$ and $d_{i, n^{\prime}}\left(s_{i}^{\prime}, s_{j}\right) \geq d_{i, n^{\prime}}\left(s_{i}, s_{j}\right)$, if Firm $i$ chooses $s_{i}^{\prime}$ instead of $s_{i}$, then its total production cost will increase by at least $c_{i} \cdot\left(d_{i, n^{\prime}}\left(s_{i}^{\prime}, s_{j}\right)-d_{i, n^{\prime}}\left(s_{i}, s_{j}\right)\right)$.

The changes in the total setup and holding costs depend on the periods in which Firm $i$ carries out its production when choosing $s_{i}^{\prime}$. Although, such periods are unknown before the corresponding $L S P$ is solved, we can estimate the cost changes by analyzing the following scenarios:

- For the lowerbound, we consider only the production cost of the additional demand resulting from the change in price in period $n^{\prime}$ without considering any potential additional holding or setup cost. This implies $c_{i} \cdot\left(d_{i, n^{\prime}}\left(s_{i}^{\prime}, s_{j}\right)-d_{i, n^{\prime}}\left(s_{i}, s_{j}\right)\right) \leq \lambda\left(s_{i}^{\prime}, s_{i}, s_{j}\right)$. By replacing equation (1) on the left-hand side of the last expression, we obtain $\underline{\lambda}\left(s_{i}^{\prime}, s_{i}\right)$.

- For the upperbound, we consider two cases:

- Given the strategy $s_{j}$, the condition $\phi\left(s_{j}, n\right)>m_{K_{i}}$ states there is a positive demand in period 1 ; thus the first scenario of the lowerbound assumes that at least one set-up occurs in interval $\left[1, n^{\prime}\right]$. In a pessimistic scenario, the last period in which a setup occurs is period 1 ; then the additional demand can be satisfied from period 1. Producing for the additional demand at the same time will lead to an additional holding cost of at least $h_{i} \cdot\left(n^{\prime}-1\right) \cdot\left(d_{i, n^{\prime}}\left(s_{i}^{\prime}, s_{j}\right)-d_{i, n^{\prime}}\left(s_{i}, s_{j}\right)\right)$. Thus, $\lambda\left(s_{i}^{\prime}, s_{i}, s_{j}\right) \leq \underline{\lambda}\left(s_{i}^{\prime}, s_{i}\right)+h_{i} \cdot\left(n^{\prime}-1\right) \cdot\left(d_{i, n^{\prime}}\left(s_{i}^{\prime}, s_{j}\right)-d_{i, n^{\prime}}\left(s_{i}, s_{j}\right)\right)$. By replacing equation (1) in the right hand side of the last expression, we obtain the first case of $\bar{\lambda}\left(s_{i}^{\prime}, s_{i}, s_{j}\right)$.

- We impose a production in period $n^{\prime}$ and potentially incur the cost of an additional setup, but avoid any holding cost.

Based on $\underline{\lambda}\left(s_{i}^{\prime}, s_{i}\right)$, we establish sufficient conditions to determine whether $s_{i}$ or $s_{i}^{\prime}$ is a better response to a given strategy of the competitor, as we show in Theorem 1. 
Theorem 1. Given the pure pricing strategies $s_{i}, s_{i}^{\prime} \in S_{i}$ such that $s_{i, n}=s_{i, n}^{\prime}, \forall n \neq n^{\prime} \wedge s_{i, n^{\prime}}^{\prime} \leq s_{i, n^{\prime}}$, if $s_{i, n^{\prime}}+s_{i, n^{\prime}}^{\prime} \leq \phi\left(s_{j}, n^{\prime}\right)+c_{i}$, then Firm $i$ prefers $s_{i}$ as a response to $s_{j}$ rather than $s_{i}^{\prime}$.

Proof. From expression (15), Firm $i$ prefers $s_{i}$ as a response to $s_{j}$ rather than $s_{i}^{\prime}$ if $R_{i}\left(s_{i}^{\prime}, s_{j}\right)-R_{i}\left(s_{i}, s_{j}\right) \leq$ $\lambda_{i}\left(s_{i}^{\prime}, s_{i}, s_{j}\right)$. The left hand side of the expression corresponds to $s_{i, n^{\prime}}^{\prime} \cdot d_{i, n^{\prime}}\left(s_{i}^{\prime}, s_{j}\right)-s_{i, n^{\prime}} \cdot d_{i, n^{\prime}}\left(s_{i}, s_{j}\right) ; \underline{\lambda}\left(s_{i}^{\prime}, s_{i}\right)$ constitutes a lower bound for the right hand side, then $s_{i, n^{\prime}}^{\prime} \cdot d_{i, n^{\prime}}\left(s_{i}^{\prime}, s_{j}\right)-s_{i, n^{\prime}} \cdot d_{i, n^{\prime}}\left(s_{i}, s_{j}\right) \leq \underline{\lambda}\left(s_{i}^{\prime}, s_{i}\right)$ establishes a sufficient condition for discarding $s_{i}^{\prime}$ as a best response to $s_{j}$. By using Lemma 1 and by replacing equation (1) in the previous expression, we obtain the following expression:

$$
-\Delta\left(s_{i}^{\prime}, s_{i}\right) \cdot\left(\alpha_{i, n^{\prime}}+\gamma_{i, n^{\prime}} \cdot s_{j, n^{\prime}}\right)-\beta_{i, n^{\prime}} \cdot\left(s_{i, n^{\prime}}^{\prime 2}-s_{i, n^{\prime}}^{2}\right) \leq c_{i} \cdot \beta_{i, n^{\prime}} \cdot \Delta\left(s_{i}^{\prime}, s_{i}\right)
$$

By dividing the previous inequality by $\beta_{i, n^{\prime}}$ we obtain the following expression (note that we re-write $\left(s_{i, n^{\prime}}^{\prime 2}-s_{i, n^{\prime}}^{2}\right)$ as $\left.-\Delta\left(s_{i}^{\prime}, s_{i}\right) \cdot\left(s_{i, n^{\prime}}+s_{i, n^{\prime}}^{\prime}\right)\right)$ :

$$
-\Delta\left(s_{i}^{\prime}, s_{i}\right) \cdot \phi\left(s_{j}, n^{\prime}\right)+\Delta\left(s_{i}^{\prime}, s_{i}\right) \cdot\left(s_{i, n^{\prime}}^{\prime}+s_{i, n^{\prime}}\right) \leq c_{i} \cdot \Delta\left(s_{i}^{\prime}, s_{i}\right)
$$

By simplifying $\Delta\left(s_{i}^{\prime}, s_{i}\right)$ from both sides of the previous inequality, we obtain the condition introduced in Theorem 1.

From Theorem 1 we derive conditions for selecting or discarding prices as part of a best response strategy, as we show in Corollary 1.

Corollary 1. Given that Firm $j$ chooses the pure pricing strategy $s_{j} \in S_{j}$, if $m_{i, K_{i}}+m_{i, K_{i}-1} \leq \phi\left(s_{j}, n\right)+c_{i}$, then the best response of Firm $i$ consists of setting the highest price for period $n$; if $m_{i, k_{i}+1}+m_{i, k_{i}} \leq$ $\phi\left(s_{j}, n\right)+c_{i}$, then the best response of Firm $i$ discards any price lower than $m_{i, k_{i}+1}$ for period $n$.

Proof. From Theorem $1, s_{i, n^{\prime}}+s_{i, n^{\prime}}^{\prime} \leq \phi\left(s_{j}, n^{\prime}\right)+c_{i}$ establishes that Firm $i$ should prefer price $s_{i, n^{\prime}}$ rather than $s_{i, n^{\prime}}^{\prime}$ in period $n^{\prime}$ when responding to $s_{j}$. The left-hand side of this expression increases with prices, which leads to two statements: (i) the maximum of that sum occurs for $s_{i, n^{\prime}}=m_{i, K_{i}}$ and $s_{i, n^{\prime}}^{\prime}=m_{i, K_{i}-1}$, so if $m_{i, K_{i}}+m_{i, K_{i}-1} \leq \phi\left(s_{j}, n\right)+c_{i}$ holds, then Firm $i$ prefers $m_{i, K_{i}}$ to any other price in period $n$; and (ii) $m_{i, k_{i}+1}+m_{i, k_{i}} \geq m_{i, k_{i}^{o}+1}+m_{i, k_{i}^{o}}$ for $k_{i}^{o}<k_{i}$, so if $m_{i, k_{i}+1}+m_{i, k_{i}} \leq \phi\left(s_{j}, n\right)+c_{i}$, then Firm $i$ discards any price lower than $m_{i, k_{i}+1}$ in period $n$.

In a similar fashion to Theorem 1 , we use $\bar{\lambda}\left(s_{i}^{\prime}, s_{i}, s_{j}\right)$ to establish adequate conditions for determining whether $s_{i}$ or $s_{i}^{\prime}$ is a better response to a particular strategy of the competitor, as we show in Theorem 2 . 
Theorem 2. Given the pure pricing strategies $s_{i}, s_{i}^{\prime} \in S_{i}$ such that $s_{i, n}=s_{i, n}^{\prime}, \forall n \neq n^{\prime} \wedge s_{i, n^{\prime}}^{\prime} \leq s_{i, n^{\prime}}$ and $s_{j} \in S_{j}$, if $s_{i, n^{\prime}}+s_{i, n^{\prime}}^{\prime} \geq \frac{\bar{\lambda}\left(s_{i}^{\prime}, s_{i}, s_{j}\right)}{\beta_{i, n^{\prime}} \cdot \Delta\left(s_{i}^{\prime}, s_{i}\right)}+\phi\left(s_{j}, n^{\prime}\right)$, then Firm $i$ prefers $s_{i}^{\prime}$ as a response to $s_{j}$ rather than $s_{i}$.

Proof. From expression (15), if $R_{i}\left(s_{i}^{\prime}, s_{j}\right)-R_{i}\left(s_{i}, s_{j}\right) \geq \lambda\left(s_{i}^{\prime}, s_{i}, s_{j}\right)$, then Firm $i$ prefers $s_{i}^{\prime}$ as a response to $s_{j}$ rather than $s_{i}$. Given that $\bar{\lambda}\left(s_{i, n^{\prime}}^{\prime}, s_{i, n^{\prime}}, s_{j}\right)$ represents an upper bound of the right-hand side of the previous expression, $R_{i}\left(s_{i}^{\prime}, s_{j}\right)-R_{i}\left(s_{i}, s_{j}\right) \geq \bar{\lambda}\left(s_{i}^{\prime}, s_{i}, s_{j}\right)$ is sufficient to discard $s_{i}$ as a best response to $s_{j}$. By replacing equation (1) in the previous expression, we obtain the condition presented in Theorem 2.

Based on Theorem 2, we derive additional conditions for selecting and discarding the prices contained in the best response strategy, as we show in Corollary 2.

Corollary 2. (a) Given that Firm $j$ chooses the pure pricing strategy $s_{j} \in S_{j}$, if $n \leq 1+\frac{m_{i, 1}+m_{i, 2}-c_{i}-\phi\left(s_{j}, n\right)}{h_{i}}$, then the best response of Firm $i$ consists of setting the lowest price for the period $n$; if $n \leq 1+$ $\frac{m_{i, k}+m_{i, k+1}-c_{i}-\phi\left(s_{j}, n\right)}{h_{i}}$, then the best response of Firm $i$ discards any price higher than $m_{i, k}$ for the pe$\operatorname{riod} n$.

(b) Given that Firm $j$ chooses the pure pricing strategy $s_{j} \in S_{j}$, if $m_{i, 1}+m_{i, 2}-c_{i}-\phi\left(s_{j}, n\right) \geq$ $\frac{f_{i}}{\beta_{i, n} \cdot\left(m_{i, 2}-m_{i, 1}\right)}$, then the best response of Firm $i$ consist of setting the lowest price for the period $n$; if $m_{i, k}+m_{i, k+1}-c_{i}-\phi\left(s_{j}, n\right) \geq \frac{f_{i}}{\beta_{i . n} \cdot\left(m_{i, k+1}-m_{i, k}\right)}$, then the best response of Firm $i$ discards any price higher than $m_{i, k}$ for the period $n$.

Proof. From Theorem 2, $s_{i, n^{\prime}}+s_{i, n^{\prime}}^{\prime} \geq \frac{\bar{\lambda}\left(s_{i}^{\prime}, s_{i}, s_{j}\right)}{\beta_{i, n^{\prime}} \cdot \Delta\left(s_{i}^{\prime}, s_{i}\right)}+\phi\left(s_{j}, n^{\prime}\right)$ establishes that Firm $i$ should prefer price $s_{i, n^{\prime}}^{\prime}$ to $s_{i, n^{\prime}}$ in period $n^{\prime}$ when responding to the pure pricing strategy $s_{j}$. The left-hand side of such condition increases with the prices, which leads to two statements: (i) the minimum of that sum occurs for $s_{i, n^{\prime}}=m_{i, 2}$ and $s_{i, n^{\prime}}^{\prime}=m_{i, 1}$, so if $m_{i, 2}+m_{i, 1} \geq \frac{\bar{\lambda}\left(s_{i}^{\prime}, s_{i}, s_{j}\right)}{\beta_{i, n^{\prime}} \cdot \Delta\left(s_{i}^{\prime}, s_{i}\right)}+\phi\left(s_{j}, n^{\prime}\right)$ holds, then Firm $i$ prefers $m_{i, 1}$ to any other price in period $n$; and (ii) $m_{i, k_{i}+1}+m_{i, k_{i}} \leq m_{i, k_{i}^{o}+1}+m_{i, k_{i}^{o}}$ for $k_{i}^{o}>k_{i}$, so if $m_{i, k_{i}+1}+m_{i, k_{i}} \geq$ $\frac{\bar{\lambda}\left(s_{i}^{\prime}, s_{i}, s_{j}\right)}{\beta_{i, n^{\prime}} \cdot \Delta\left(s_{i}^{\prime}, s_{i}\right)}+\phi\left(s_{j}, n^{\prime}\right)$, then Firm $i$ discards any price higher than $m_{i, k_{i}}$ in period $n$.

If now we take the second scenario of $\bar{\lambda}\left(s_{i}^{\prime}, s_{i}, s_{j}\right)$ in Lemma 1 into consideration, we can derive part (b) of the corollary in a similar fashion.

We can use the results of Corollary 1 and Corollary 2 to reduce the number of binary variables of the best response formulation. Therefore, when we are certain that the best response to the competitor's pricing strategy consists in setting price $m_{i, k}$ in period $n$, the decision for that period is avoided. When we are certain that the best response to the competitor's pricing strategy discards prices lower than $m_{k}$ in period $n$, we add the constraint $w_{i, k^{\prime}, n}=0, \forall k^{\prime}<k$ in model (12)- (14). When we are certain that the 
best response to the competitor's pricing strategy discards prices higher than $m_{i, k}$ in period $n$, we add the constraint $w_{i, k^{\prime}, n}=0, \forall k^{\prime}>k$ in model (12)- (14).

Example 3. Consider Firm $i$ and Firm $j$ of our example. Table 2 summarizes the parameters of Corollary 1, where row $\alpha_{i, n}=5$ corresponds to period 1,3 and 4, and $\alpha_{i, n}=10$ corresponds to period 2.

\begin{tabular}{cccccc}
\hline \multirow{2}{*}{$\alpha_{i, n}$} & $m_{i, 1}+m_{i, 2}$ & $m_{i, 2}+m_{i, 3}$ & \multicolumn{3}{c}{$\phi\left(s_{j}, n^{\prime}\right)+c_{i}$} \\
\hline 5 & 7 & 9 & 6 & 6.5 & 7 \\
10 & 7 & 9 & 11 & 11.5 & 12 \\
\hline
\end{tabular}

Table 2: Data for Corrolary 1 for Firm $i$

From Table 2, we observe that $m_{i, 1}+m_{i, 2} \leq \phi\left(s_{j}, n^{\prime}\right)+c_{i}$ for $\alpha_{i, n}=5$ and $s_{j, n^{\prime}}=4$, thus we discard price any pure pricing strategy that contains the price 3 in periods 1,3 or 4 from the set of potential best responses. Note that for period 2, the condition of the Corollary 1 are not met, so no price can be discarded.

Table 3 shows similar analysis for Firm $j$. In this case, we discard any pure pricing strategy that contains the price 2 from the set of potential best responses.

\begin{tabular}{cccccc}
\hline \multirow{2}{*}{$\alpha_{j, n}$} & $m_{j, 1}+m_{j, 2}$ & \multirow{2}{*}{$m_{j, 2}+m_{j, 3}$} & \multicolumn{4}{c}{$\phi\left(s_{i}, n^{\prime}\right)+c$} \\
& & $s_{i, n^{\prime}}=3$ & $s_{i, n^{\prime}}=4$ & $s_{i, n^{\prime}}=5$ \\
\hline 4 & 5 & 7 & 5.5 & 6 & 6.5 \\
\hline
\end{tabular}

Table 3: Data for Corrolary 1 for Firm $j$

\subsection{Reactive Pruning}

Next, we determine whether $s_{i}$ is the best response to $s_{j}^{\prime}$, given that $s_{j}^{\prime}$ results from increasing (or decreasing) the price in one period with respect to $s_{j}$ and $s_{i}$ is the best response to $s_{j}$. We start by proposing upper and lower bounds for $\tau\left(s_{j}^{\prime}, s_{i}, s_{j}\right)$ in Lemma 2 .

Lemma 2. Given the competitor's pure pricing strategies $s_{j}, s_{j}^{\prime} \in S_{j}$ such that $s_{j}(n)=s_{j}^{\prime}(n), \forall n \neq$ $n^{\prime} \wedge s_{j}\left(n^{\prime}\right)<s_{j}^{\prime}\left(n^{\prime}\right)$ and let $\tilde{n}$ be the last period of interval $\left[1, n^{\prime}\right]$ in which Firm $i$ produces when implementing the best response to $s_{j}, \underline{\tau}\left(s_{j}^{\prime}, s_{j}\right)$ and $\bar{\tau}\left(s_{j}^{\prime}, s_{i}, s_{j}\right)$ constitute lower and upper bounds for $\tau\left(s_{j}^{\prime}, s_{i}, s_{j}\right)$, respectively, where

$$
\underline{\tau}\left(s_{j}^{\prime}, s_{j}\right)=c_{i} \cdot \gamma_{i, n^{\prime}} \cdot \Delta\left(s_{j}, s_{j}^{\prime}\right)
$$


and

$$
\bar{\tau}\left(s_{j}^{\prime}, s_{j}\right)=\underline{\tau}\left(s_{j}^{\prime}, s_{j}\right)+\min \left\{h_{i} \cdot\left(n^{\prime}-\tilde{n}\right) \cdot \gamma_{i, n^{\prime}} \cdot \Delta\left(s_{j}, s_{j}^{\prime}\right), f_{i}\right\},
$$

Proof. Since $d_{i, n}\left(s_{i}, s_{j}\right)=d_{i, n}\left(s_{i}, s_{j}^{\prime}\right), \forall n \neq n^{\prime}$ and $d_{i, n^{\prime}}\left(s_{i}, s_{j}\right) \leq d_{i, n^{\prime}}\left(s_{i}, s_{j}^{\prime}\right)$, if the competitor changes its strategy from $s_{j}$ to $s_{j}^{\prime}$, the total unit production cost of Firm $i$ will increase by at least $c_{i} \cdot\left(d_{i, n^{\prime}}\left(s_{i}, s_{j}^{\prime}\right)-\right.$ $\left.d_{i, n^{\prime}}\left(s_{i}, s_{j}\right)\right)$. In an optimistic scenario, the setup and holding costs will remain identical to those obtained when responding to $s_{j}$; so, the increase of the total unit production cost is a lower bound for $\tau\left(s_{j}^{\prime}, s_{i}, s_{j}\right)$. We obtain $\underline{\tau}\left(s_{j}^{\prime}, s_{j}\right)$ by replacing equation $(1)$ in $c_{i} \cdot\left(d_{i, n^{\prime}}\left(s_{i}, s_{j}^{\prime}\right)-d_{i, n^{\prime}}\left(s_{i}, s_{j}\right)\right)$.

We now estimate the changes in setup and holding costs for less optimistic scenarios. A first alternative is to increase the production of $\tilde{n}$; the number of setups does not increase, so the total cost increases by $\underline{\tau}\left(s_{j}^{\prime}, s_{j}\right)+h_{i} \cdot\left(n^{\prime}-\tilde{n}\right) \cdot \gamma_{i, n^{\prime}} \cdot \Delta\left(s_{j}, s_{j}^{\prime}\right)$. A second alternative is to produce in period $n^{\prime}$ to meet the additional demand in that period. The holding cost does not vary, but a new set-up occurs, so the total cost increases by $\underline{\tau}\left(s_{j}^{\prime}, s_{j}\right)+f_{i}$. Both cases provide upper bounds for $\tau\left(s_{j}^{\prime}, s_{i}, s_{j}\right)$.

Once we obtain the best response to a competitor's pure pricing strategy, we can use Lemma 2 to obtain upper and lower bounds for the best response when the competitor's varies its price in one period, as we show in Theorem 3 .

Theorem 3. Let $\tilde{n}$ be the last period of interval $\left[1, n^{\prime}\right]$ in which Firm i produces when implementing the best response to $s_{j} \in S_{j}$, (a) if the competitor increases its price in period $n^{\prime}$ with respect to $s_{j}$, then the price of the new best response in period $n^{\prime}$ is greater than or equal to $s_{i, n^{\prime}}^{*}\left(s_{j}\right)-h_{i} \cdot\left(n^{\prime}-\tilde{n}\right)$; (b) if the competitor decreases its price in period $n^{\prime}$ with respect to $s_{j}$, then the price of the new best response in period $n^{\prime}$ is less than or equal to $s_{i, n^{\prime}}^{*}\left(s_{j}\right)+h_{i} \cdot\left(n^{\prime}-\tilde{n}\right)$.

Proof. Given $s_{j}$, let $s_{j}^{\prime}$ be the pure pricing strategy resulting from increasing the price in period $n^{\prime}$; Firm $i$ will prefer $s_{i}^{*}\left(s_{j}\right)$ to any $s_{i} \in S_{i}$ as a response to $s_{j}^{\prime}$ when condition $B_{i}\left(s_{i}^{*}\left(s_{j}\right), s_{j}^{\prime}\right) \geq B_{i}\left(s_{i}, s_{j}^{\prime}\right)$, holds. We can re-write the previous expression as follows.

$$
\begin{array}{r}
B_{i}\left(s_{i}^{*}\left(s_{j}\right), s_{j}\right)+\gamma_{i, n^{\prime}} \cdot \Delta\left(s_{j}, s_{j}^{\prime}\right) \cdot\left(s_{i, n^{\prime}}^{*}\left(s_{j}\right)-s_{i, n^{\prime}}\right) \geq \\
B_{i}\left(s_{i}, s_{j}\right)+\tau\left(s_{j}^{\prime}, s_{i}^{*}\left(s_{j}\right), s_{j}\right)-\tau\left(s_{j}^{\prime}, s_{i}, s_{j}\right) .
\end{array}
$$

From Definition $1, B_{i}\left(s_{i}^{*}\left(s_{j}\right), s_{j}\right) \geq B_{i}\left(s_{i}, s_{j}\right), \forall s_{i} \in S_{i}$. Hence, expression (19) is a sufficient condition for satisfying expression (18). 


$$
\gamma_{i, n^{\prime}} \cdot \Delta\left(s_{j}, s_{j}^{\prime}\right) \cdot\left(s_{i}^{*}\left(s_{j}\right)-s_{i, n^{\prime}}\right) \geq \tau\left(s_{j}^{\prime}, s_{i}^{*}\left(s_{j}\right), s_{j}\right)-\tau\left(s_{j}^{\prime}, s_{i}, s_{j}\right) .
$$

Moreover, Lemma 2 establishes that $\bar{\tau}\left(s_{j}^{\prime}, s_{j}\right) \geq \tau\left(s_{j}^{\prime}, s_{i}^{*}\left(s_{j}\right), s_{j}\right)$ and $\underline{\tau}\left(s_{j}^{\prime}, s_{j}\right) \leq \tau\left(s_{j}^{\prime}, s_{i}\left(s_{j}\right), s_{j}\right)$, thus $\bar{\tau}\left(s_{j}^{\prime}, s_{j}\right)-\underline{\tau}\left(s_{j}^{\prime}, s_{j}\right)$ is an upper bound for the right-hand side of expression (19). So, expression (20) is a sufficient condition for (19).

$$
\gamma_{i, n^{\prime}} \cdot \Delta\left(s_{j}, s_{j}^{\prime}\right) \cdot\left(s_{i, n^{\prime}}^{*}\left(s_{j}\right)-s_{i, n^{\prime}}\right) \geq h_{i}\left(n^{\prime}-\tilde{n}\right) \cdot \gamma_{i, n^{\prime}} \cdot \Delta\left(s_{j}, s_{j}^{\prime}\right)
$$

By reorganizing expression (20), we obtain $s_{i, n^{\prime}}^{*}\left(s_{j}\right)-h_{i} \cdot\left(n^{\prime}-\tilde{n}\right) \geq s_{i, n^{\prime}}$. The proof for the part (b) of the theorem is derived in a similar fashion.

As for the active pruning, we can use the results of Theorem 3 to reduce the number of binary variables used in the best response problem. Furthermore, the reactive pruning may provide a new best response based on a subset of already computed best responses. We may therefore calculate the pricing equilibrium between firms by performing a partial revision of the competitor's pure pricing strategies, as we explain in Section 5.

\section{Obtaining a Nash Equilibrium}

In this section we explain how to implement the models and the active and reactive pruning introduced in Section 3 and Section 4 to reduce the computations for price equilibrium between firms. We propose and compare three approaches for constructing the payoff matrices: Best Response (Approach $B R$ ), Active Pruning (Approach $A P$ ) and Full Pruning (Approach FP). The three approaches provide the same pricing equilibrium, but the computational time may vary between them. Here, we describe each approach.

- Approach $B R$ limits the payoff mapping to the best responses for each competitor's pure pricing strategy. We use model (12) - (14) to compute these best responses. Based on the partial payoff mapping that contains only best responses, we use formulation (6) - (11) to compute the equilibrium.

- Approach $A P$ is similar to Approach $B R$, but now we take the results of Corollaries 1 - 2 into account. Thus, before solving model (12) - (14), we set the value of certain binary variables either to 0 or 1 according to the conditions derived from those corollaries. Then, we may reduce the time for getting the solution of this model. 
- Approach FP also includes the conditions derived in Corollaries 1 - 2, but in addition it takes the reactive pruning derived of Theorem 3 into account when we explore the competitor's pure pricing strategies.

Note that, the computations involved in Approach FP depends on the sequence used to revise the competitor's pure pricing strategies. We propose a sequence that increases the number of prices that can be pruned when solving the best response problem. In order to illustrate the dynamics of the proposed sequence, we use Figure 1a to represent such sequence when the problem consists of two periods $(N=2)$ and the firms can choose the pricing from a set consisting of three prices $\left(K_{i}=K_{j}=3\right)$. Each cell symbolizes the competitor's pure pricing strategy, where $[a, b]$ denotes the price selected by the competitor for period 1 and period 2, and the number between '()'represents the position in the sequence of revision. Our sequence consists of the following steps.

1. We start by solving the best response problem for the scenario in which the competitor sets the lowest price in each period, i.e., we obtain $s_{i}^{*}\left(s_{j}\right) \mid s_{j, n}=m_{j, 1}, \forall n$. Based on $s_{i}^{*}\left(s_{j}\right)$, we establish lower bounds for the best responses when the competitor increases the price in a single period. In our example, we start the revision for the cell containing the number '(1)'. Once we solve the best response problem for that cell, we can set lower bounds for the best response problems of cells '(2)', '(3)', '(4)'and '(7)'(red dashed lines).

2. We solve the best response problem when the competitor increases the price to its highest value in period 1, i.e., we obtain $s_{i}^{*}\left(s_{j}\right) \mid s_{j, n}=m_{j, 1}$, for $n \neq 1 \wedge s_{j, 1}=m_{j, K_{j}}$. Based on that response, we establish upper bounds for the best responses to the competitor's strategies where the price of period 1 is decreased, and lower bounds for the best responses when the competitor increases the price of another period. In our example, we continue revising the cell with the number '(2)'. Note that, when solving the best response problem for that cell we take into consideration the lower bound we established in Step 1. Based on the obtained solution, we set lower bounds for the best response problems of cells '(8)'and '(5)'(red dashed line), and upper bounds for the best response problems of cell '(3)'(green dashed lines).

3. We solve the best response problem when the competitor sets a "medium" price in period 1, i.e., we obtain $s_{i}^{*}\left(s_{j}\right) \mid s_{j, n}=m_{j, 1}$, for $n \neq 1 \wedge s_{j, 1}=m_{j,\left\lceil\frac{K_{j}}{2}\right\rceil}$. For solving the problem, we take the already calculated lower and upper bounds into account. In the same fashion as in Steps 1 and 2, we establish upper and lower bounds for other best responses. In our example, we solve the 
best response problem of cell '(3)'by including the lower bound obtained in Step 1 and the upper bound of Step 2. Based on the obtained solution, we determine lower bounds for the best response problems of cells '(6)'and '(9)'. For the case $K_{i}>3$, the step continues by setting the price in period 1 to the two "medium" prices between $m_{j, 1}$ and $m_{j,\left\lceil\frac{K_{j}}{2}\right\rceil}$, and $m_{j,\left\lceil\frac{K_{j}}{2}\right\rceil}$ and $m_{j, K_{j}}$. We repeat this procedure until we have revised all the prices of period 1.

Step 4 and 5 should be performed sequentially for periods $\hat{n}=2 \ldots, N$.

4. We revise the strategy in which the competitor sets the highest price in period $\hat{n}$, but the rest of the prices remains in their lowest value, that is, we obtain $s_{i}^{*}\left(s_{j}\right) \mid s_{j, n}=m_{j, 1}$, for $n \neq \hat{n} \wedge s_{j, \hat{n}}=m_{j, K_{j}}$. We repeat step 1 and 2 , but we substitute period 1 by period $\hat{n}$. In our example, we solve the best response problems of cells '(4)', '(5)', and '(6)'.

5. We calculate the best response problems for the competitor's pricing strategy corresponding to the lowest price for period $\hat{n}$, and later for all the resulting "medium"prices until we have revised all the prices of period $\hat{n}$. In our example, we solve the best response problems of cells '(7)', '(8)', and '(9)'.
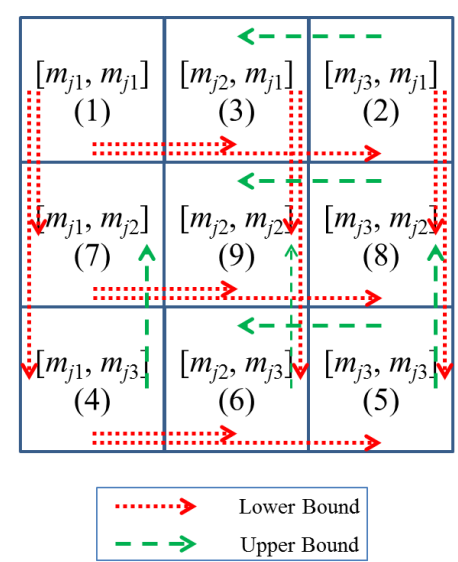

(a) Proposed Sequence.
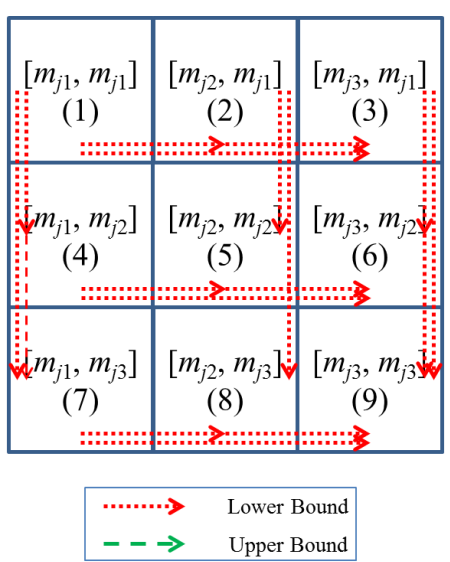

(b) Ordinal Sequence.

Figure 1: Sequence for revising pure pricing strategies of the competitor.

It is interesting to point out that the proposed sequence allows to solve some competitor's pure pricing strategies when upper and lower bounds are already calculated (e.g. when solving the pricing strategy $\left[m_{j, 1}, m_{j, 2}\right]$ of Figure 1a). If the bounds are tight, the best response arises from such bounds, and consequently, there is not need for solving the corresponding best response problem. This advantage does not occur when we revise the competitor's pure pricing strategies in an ordinal sequence, as we show in Figure 1b. Hence, our sequence reduces the computations for a pricing equilibrium rather than the 
ordinal sequence.

\section{Comparison of solution methods}

In this section we investigate the efficiency of the approaches introduced in Section 5. In particular, we aim to get insights about the following aspect of the $L S P$ under price competition:

- The computational time for obtaining payoff matrices by implementing the Approach $B R$, Approach $A P$ and Approach FP.

- The sensitivity of the computational time to the length of the horizon and the number of possible prices.

Our experiments consider identical firms, i.e., the operations, the set of available prices and the demand functions are characterized by identical parameters for both firms. We derive the NE that maximizes the sum of the profit of the firms, in other words, we maximize $\max \left(\bar{b}_{i}+\bar{b}_{j}\right)$ subject to the constraints $(6)$ (11). If such equilibrium does not exist, then we compute an MNE. We implemented the tests in the Java language. The computer used was a 6 -Core Intel Xeon $2 \times 2.66 \mathrm{GHz}$ with $48 \mathrm{~GB}$ of RAM. MIP problems are solved using Gurobi 4.6.1 (http://www.gurobi.com/).

\subsection{Instance setting}

In order to cover a broad spectrum of operational, pricing and demand scenarios, we analyze 1,792 instances, that result from the full factorial combination of the following values of the parameters of the problem. We represent the operations of the firms by: $N=2, \ldots, 7 ; f_{i}=\{2.00,8.00\}$ and $h_{i}=\{0.20,1.00\}$. Without loss of generality, we set the unit production cost of the items to 0 , indeed, we can assume that the set of prices already discounted this cost. We set the number of prices that firms can choose in each period to $K_{i}=3$. Moreover, we consider two more parameters when setting the prices of an instance: the lowest price $\left(m_{i, 1}\right)$ and the difference between two consecutive prices $\left(d p_{i}\right)$. We study instances in which $m_{i, 1}=\{1,2\}$ and $d p_{i}=\{1,2\}$. In terms of the demand function, we vary the sensitivity parameters as follows: $\beta_{i, n}=\{0.66,2.66\} ; \gamma_{i, n}=\left\{0.20 \cdot \beta_{i, n}, 0.80 \cdot \beta_{i, n}\right\} ;$ note that, it is natural to assume that $\gamma_{i, n} \leq \beta_{i, n}$ since in general the demand for a product is more sensitive to its own price. Further, we use parameter $\underline{d}=\{0.40,1.60\}$ for representing the minimum demand in each period; so, any pair of pricing strategies leads to non negative demand. 


\subsection{Computational time}

We compare the computational time when implementing Approach $B R$, Approach $A P$ and Approach FP. Our initial concern lies on measuring how the computational time grows with the length of the horizon. Table 4 summarizes the average computational time for calculating payoff matrices when implementing the approaches studied. We include a column representing the number of pricing strategies revised when implementing the corresponding approach. Thus, the values on that column for Approach $B R$ correspond to twice the number of pricing strategies of the competitor, i.e. $2 \cdot K_{i}^{N}$ (we multiply by two because the problem is solved for the two firms). If we use a full inspection of the combination of the pricing strategies of the firms such values increases to $K_{i}^{2 N}$. Therefore, implementing a full inspection is pointless as the value of $N$ increases. Further, the last column of Table 4 represents the average time for computing the NE (or MNE) based on constraints (6) - (11).

\begin{tabular}{|c|c|c|c|c|c|c|c|}
\hline \multirow[b]{3}{*}{$N$} & \multicolumn{6}{|c|}{ Approach } & \multirow{3}{*}{$\begin{array}{c}\text { NE } \\
\text { Time }\end{array}$} \\
\hline & \multicolumn{2}{|r|}{$\mathrm{BR}$} & \multicolumn{2}{|c|}{$\mathrm{AP}$} & \multicolumn{2}{|r|}{ FP } & \\
\hline & Time & Rev. strategies & Time & Rev. strategies & Time & Rev. strategies & \\
\hline 2 & 0.10 & 18 & 0.04 & 4.2 & 0.01 & 1.50 & 0.00 \\
\hline 3 & 0.34 & 54 & 0.16 & 10.5 & 0.03 & 6.7 & 0.00 \\
\hline 4 & 1.73 & 182 & 0.83 & 74.6 & 0.11 & 42.8 & 0.01 \\
\hline 5 & 9.31 & 486 & 6.19 & 245.6 & 0.59 & 130.0 & 0.01 \\
\hline 6 & 80.32 & 1,458 & 29.84 & 770.8 & 2.96 & 392.2 & 0.06 \\
\hline 7 & 176.18 & 4,374 & 108.26 & $2,398.4$ & 15.68 & $1,070.6$ & 0.52 \\
\hline
\end{tabular}

Table 4: Average Computational Time (in seconds) and Average Revised Pricing Strategies for Different Approaches and Different Horizon Lengths.

The first column of Table 4 provides evidence of the exponential growth of the computational time of Approach $B R$ as the number of periods increases. Such time is significant in comparison to the one needed for obtaining NE from the resulting payoff matrix (last column in Table 4), thus any effort for speed up the pricing problem under competition should focus on the matrix construction. In this sense, implementing Approach AP reduces the computational time because such approach shrinks the space of potential best responses to the competitor's pure pricing strategies. However, the most remarkable result exposed in Table 4 is the significant time reduction when taking into consideration the solution of the already revised best response problems (Approach FP); computing a full payoff matrix requires revising a fraction of the full set of pure pricing strategies. Although Approach FP may not be able to determine some of the best responses in advance, it reduces the time for computing the best pricing, because the space of feasible solutions is shrunk by including the constraints we derived in Section 4 . 
Further, we study the robustness of the time savings due to Approach FP across different instances of the problem. With this in mind, we analyze the distribution of the computational time. In particular, me measure the time for solving each instance for Approach $B R$, Approach AP and Approach FP. Figure 2 shows the distribution of the computational time of Approach $A P$ and Approach $F P$ as a percentage of the computational time of Approach $B R$. In this figure we present the results in a box and whiskers format: the upper and the lower bounds of the boxes represent the percentile 25 and 75 of the percentages, respectively; the upper and the lower whiskers correspond to the percentile 5 and 95 of the percentages; the markers are the average values obtained for the relative running times.

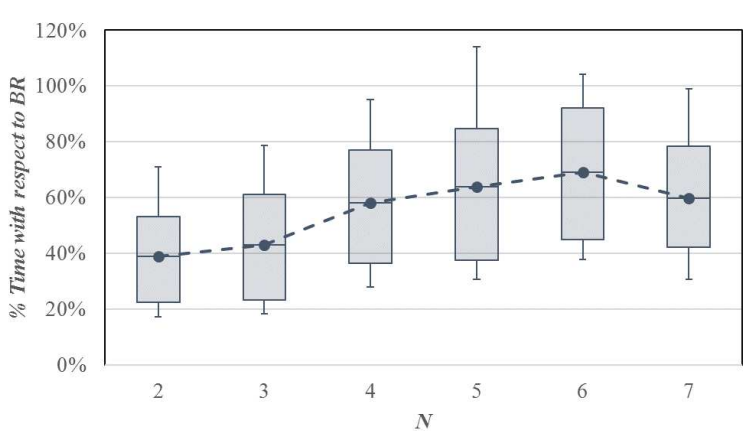

(a) Approach $A P$.

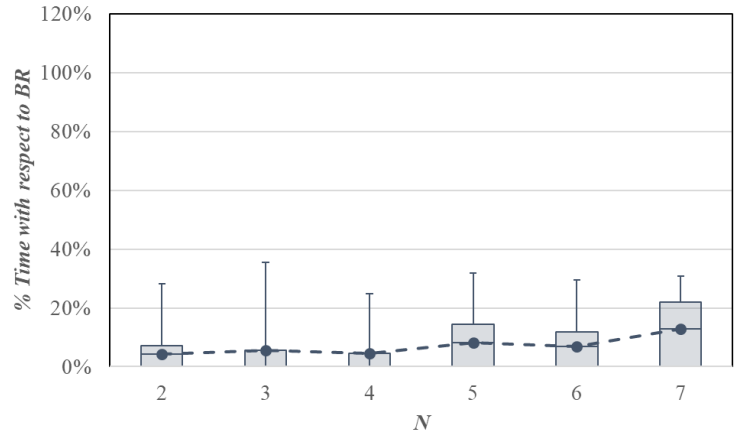

(b) Approach FP.

Figure 2: Computational Time Savings in Comparison to Approach FP.

Figure 2a shows that the number of instances where Approach $B R$ outperforms Approach $A P$ increases with the horizon length. This means that the additional computation for discarding prices of Approach $A P$ does not compensate the computational time of implementing that approach. A different pattern is observed for Approach FP. Figure 2b shows that the computation time speed up factor is not affected by the problem size, because the additional computations required for implementing the lower and upper bounds of Approach FP are compensated by the fact that many best response problems do not need to be solved. Given the clear dominance of implementing Approach FP in comparison to Approach $A P$, in the rest of the analysis of this paper we omit the results for that last approach.

Because measuring the computational time of Approach $B R$ is quickly becoming prohibitive for long horizons, we estimate how fast each approach revises and solves the best response problem. Thus, for the instances studied we run each approach for 10 seconds and we compute the number of solved best response problems as a percentage of the total number of competitor's pure pricing strategies that need to be considered, from hereon denoted $\%$ rs. Table 5 shows the average values of $\%$ rs in our experiments. This table underscores the limitations of Approach $B R$ for addressing larger instances and how Approach 
FP can construct a big portion of the payoff matrix in a short time.

Based on the values of \%rs we can forecast the time it will take to fulfill the payoff matrices of the firms. With this in mind, we use $\frac{10}{60 \% \text { rs }}$ to estimate the time (in minutes) for full-filling the payoff matrices. Figure 3 summarizes the estimated time in our experiments. As we can observe, Approach $B R$ results in prohibitive computational times as the size of the instance grows. Approach $F P$ is highly efficient in reducing such time; for instance, when the horizon consists of 12 periods the expected time for implementing Approach $B R$ is 879 minutes, that is significantly higher than the time for Approach FP (2.24 minutes). Note that, the way we compute the expected time represents a pessimistic scenario for Approach FP, because this approach saves time from the information that we can extract from already appraised best response problems, this is not taken into account in our simple extrapolation of the computational time, and therefore, we can expect even faster computational times in practice.

\begin{tabular}{|c|c|c|}
\hline$N$ & Approach $B R$ & Approach $F P$ \\
\hline 7 & $14.25 \%$ & $43.25 \%$ \\
\hline 8 & $2.49 \%$ & $36.64 \%$ \\
\hline 9 & $0.68 \%$ & $32.06 \%$ \\
\hline 10 & $0.16 \%$ & $25.19 \%$ \\
\hline 11 & $0.07 \%$ & $22.90 \%$ \\
\hline 12 & $0.02 \%$ & $7.43 \%$ \\
\hline
\end{tabular}

Table 5: Average Percentage of Revised Pricing Strategies in 10 Seconds For Long Horizons Lengths

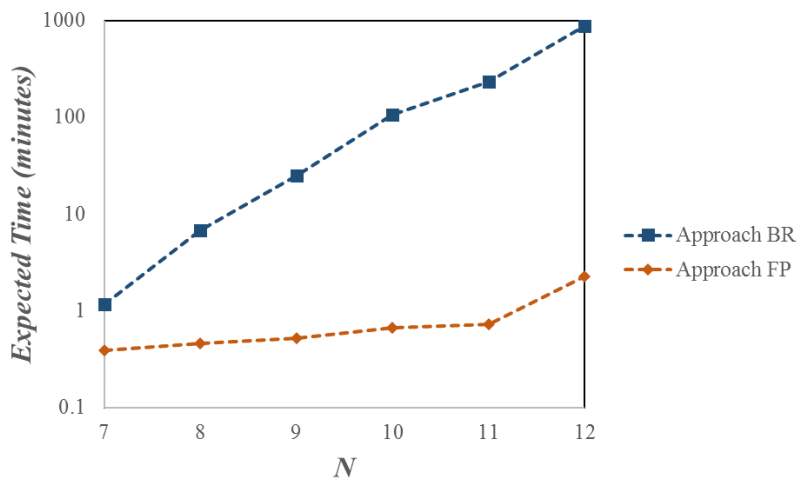

Figure 3: Estimated Computational Time for Computing Payoff Matrices For Long Horizons Lengths.

Further, we compare the computational times when implementing Approach $B R$ and Approach $F P$ for different sizes of the set of available prices. In our experiments we consider the instances defined above, but now we vary the number of available prices in the range $K_{i}=\{2,3,4\}$, and we set the horizon to $N=6$ periods. Thus, this experiment consists of solving 144 instances for each value of 
$K_{i}=\{2,3,4\}$. Table 6 summarizes the average computational time for calculating the payoff matrices in our experiment. For each approach we include a column representing the number of pricing strategies considered when implementing the corresponding approach. Again, Approach FP reports significant time savings in comparison to Approach $B R$.

\begin{tabular}{ccccccc}
\hline & \multicolumn{4}{c}{ Approach } & \\
\cline { 2 - 3 }$K_{i}$ & Time & Rev. strategies & & Time & Rev. strategies & TE \\
\hline 2 & 0.46 & 128 & & 0.03 & 10.5 & \\
\hline 3 & 29.46 & 1,458 & & 3.56 & 165.3 & 0.02 \\
4 & 453.83 & 8,192 & & 4.25 & 294.14 & \\
\hline
\end{tabular}

Table 6: Average Computational Time (in seconds) and Average Revised Pricing Strategies for Different Approaches and Different Cardinality of Set of Potential Prices.

\section{Managerial Insights}

In this section we show the potential usefulness of our model through illustrative examples of competitive situations that might arise in real life. In particular we look at the following questions.

- The effect of implementing a constant price policy on the profits of the firms. Our analysis provides evidence about the potential gains due to dynamic pricing.

- The effect of incorporating competitor's decision on the decision making of a firm.

We will start by using our example to illustrate the dynamics that result from dynamic pricing and lot sizing when one of the firms faces a demand surge.

Example 4. Consider Firm $i$ and Firm $j$ of Example 1 , but now assume $\alpha_{i, 2}=10$, i.e. Firm $i$ faces a peak of demand in the second period of the horizon. If both firms do not change the pricing strategies they choose in Example 1, the profit of Firm $i$ will be 53 and the profit of Firm $j$ will not vary. Nevertheless, the Firm $i$ can use the fact that its demand tends to be higher in period 2, consequently, the pricing and planning of the firms may vary. Thus, the pair of pure pricing strategies $s_{i}=\{3,5,4,4\}$ and $s_{j}=\{3,4,3,4\}$ constitutes a NE. Given the new pricing, the demands faced by the firms are $d_{i}=$ $\{3.5,7.0,2.5,3.0\}$ and $d_{j}=\{2.5,2.5,3.0,2.0\}$. Thus, the production planning of both firms vary with respect to Example 1: $x_{i}=\{3.5,9.5,0.0,3.0\}$ and $x_{j}=\{5.0,0.0,5.0,0.0\}$. The new profits are $B_{i}\left(s_{i}, s_{j}\right)=$ 56.0 and $B_{j}\left(s_{i}, s_{j}\right)=24.0$, which are higher than the profits that both firms can obtain by keeping the pricing strategies of Example 1. 


\subsection{Static versus Dynamic pricing}

We analyze how a dynamic pricing strategy can increase the profits of the firm in comparison to setting a static price for the whole horizon. To do so, we compute the profit of a firm for three types of experiments: (i) both competitors set static pricing strategies; (ii) both competitors set dynamic pricing strategies; (iii) the firm for whom we estimate profits sets a static pricing strategy and its competitor sets dynamic pricing. We use $b_{S S}, b_{D D}$ and $b_{S D}$ to denote the profits obtained by a firm for experiments (i), (ii), and (iii), respectively. Note that, $b_{S D}$ corresponds to the natural benchmark for dynamic pricing strategies, because a firm can control its own strategy, but it cannot influence on the type of strategy of its competitor. Nevertheless, we also compare dynamic pricing strategies with $b_{S S}$, even though implementing simultaneously static pricing strategies requires coordinated pricing between competitors, that in realbusiness environments may be penalized as a collusive initiative.

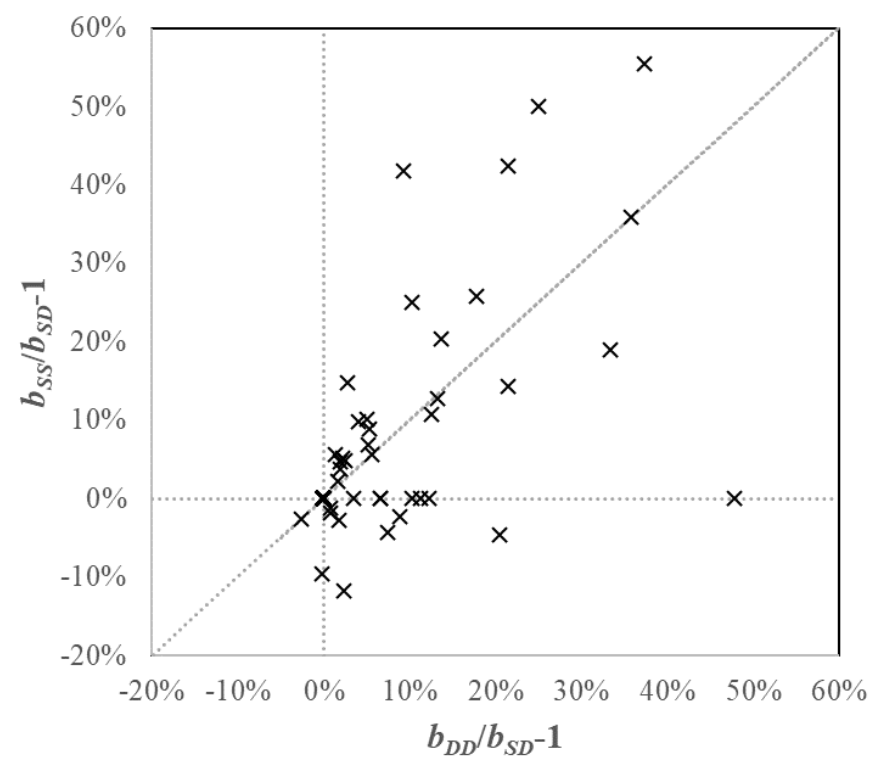

Figure 4: Profits for Dynamic and Static Pricing Stategies.

Figure 4 shows the results of our experiments for the instances used in this section and the horizon $N=6$. The x-axis of Figure 4 represents the percentage of increase of profits when implementing dynamic pricing strategies in comparison to setting a static price for the whole horizon (when competitor may implement a dynamic pricing strategy). As we can observe from the figure, in almost all the instances that we tested (just with the exception of one), a firm increases its profit when setting a dynamic pricing strategy. On average, the dynamic pricing strategy increases the profit of a firm on $4.9 \%$ in comparison to a static pricing strategy. The y-axis of Figure 4 represents the percentage of increase of profits when 
both competitors set static pricing strategies in comparison to setting a static price for the whole horizon (when competitor may implement a dynamic pricing strategy). As we can observe from the figure, for certain instances the static pricing strategies may lead to larger profits than dynamic pricing strategies (crosses above the dashed line), but again, the result is a theoretical exercise because in practice the simultaneous static pricing may be infeasible. Moreover in many instances setting a static pricing may hurt the firm if the competitor changes to a dynamic pricing strategy (crosses belox the horizontal axis).

\subsection{Effect of modeling competition}

Our analysis continues by measuring the impact of addressing competitor's decisions into the pricing and production/inventory planning. To do so, we compute the profits of Firm 1 when this firm makes pricing and production/inventory planning without taking its competitor's decisions into account. In order to model the demand function of Firm 1, we fix the values of the competitor's prices on equation (1), and afterwards, we solve the corresponding best response problem. Contrary to Firm 1, we assume that Firm 2 addresses the decisions of Firm 1. We model the pricing and planning of Firm 2 by solving the best response problem to the pricing strategy of Firm 1. Note that, even though Firm 1 overlooks competitors' decisions, its actual revenues depends on the demand derived from the pricing strategies of both firms. Moreover, the actual cost of Firm 1 is derived from the observed demand and the already planned production/inventory planning.

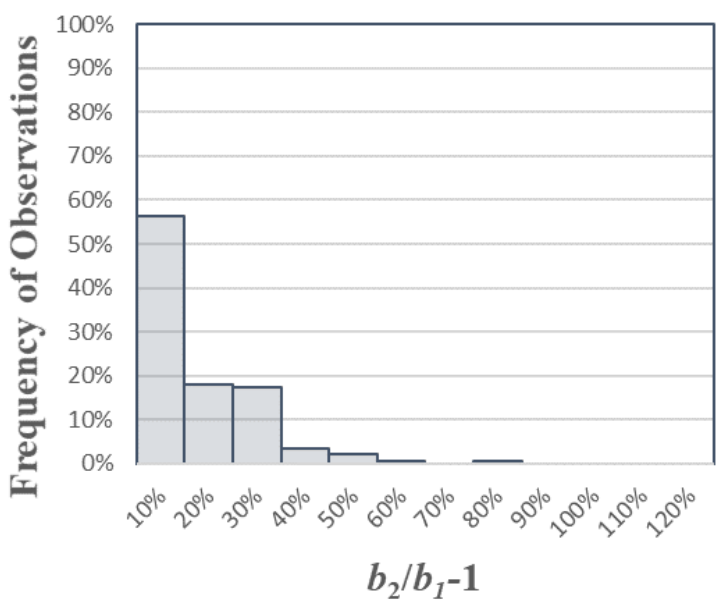

(a)

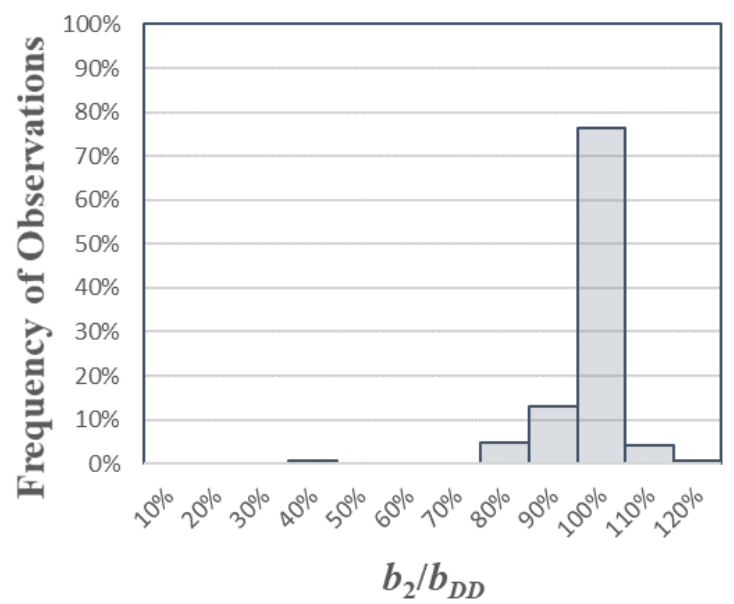

(b)

Figure 5: Effect of Modelling Competition.

Figure 5 summarizes the results for Firm 1 and Firm 2 for the instances used in this section and the horizon $N=6$. In order to represent the decisions of Firm 1, we randomly set its competitor's price. 
In Figure 5a we provide a histogram of the observed gains of Firm $2\left(b_{2}\right)$ in comparison to Firm $1\left(b_{1}\right)$. From the figure we get evidence that missing competitor's decisions can significantly hurt the profits of the firms; on average, the profits of Firm 2 are 12.7\% higher than for Firm 1 in our experiments. In Figure $5 \mathrm{~b}$ we provide a histogram of the observed ratio between the profit of Firm $2\left(b_{2}\right)$ in comparison to the profits obtained when both firms consider competitor's actions $\left(b_{D D}\right)$. The figure shows that taking competitor's decisions into account leads to revenues whose magnitude does not vary significantly with how the competitors makes decisions; on average, $b_{2}$ capture $96.0 \%$ of the profits derived from $b_{D D}$. Moreover, in some instances, Firm 2 gain from the fact that the competitor has a myopic pricing strategy in such a way the profits are even higher than $b_{D D}$.

\section{Summary and Conclusions}

We study the joint pricing and production/inventory planning when two firms manufacture and sell mutually substitutable products. Our modelling is based on two premises: there is a discrete set of prices firms can use for each period; and the production/inventory decisions of each firm can be modelled by an LSP. Even though we can solve a sequence of MIP models for finding the pricing strategies that constitute an NE, the computation of such an equilibrium requires the full mapping of the firms' payoffs, which increases exponentially with the length of the horizon and the number of available prices. We propose a method that discards pricing strategies not leading to an NE before computation. Our method consists of two steps. In the first, we characterize the pricing strategies of a firm that constitute the set of best responses to the competitor's strategies. In the second, we calculate the NE for the pricing strategies obtained in the previous step. Our numerical experiments show that computational time is significantly reduced by the implementation of our method rather than a full mapping of the firms' payoffs.

Although our findings are limited to two competitors, our method can easily be extended to scenarios with more competitors. Indeed, we could expect that the savings in computational time become even more significant in such scenarios, due to the exponential increase of the potential combinations of prices. Moreover, even though we assume a linear relationship between demand and prices, our method suits other types of demand functions.

One of the main contributions of this paper is that it reduces the decision-making cycle between Marketing and Operations departments. When these departments make their decisions independently of each other, inefficiency arises because the operations department may not be able to produce what marketing offers the customers, and/or the pricing leads to revenues that do not cover production costs. 
In order to reduce such inefficiency, a cycle of information and decision-making is required across the firm's departments, but this cycle can result in additional work and slower response to customers. Given that we propose a joint approach for pricing and operational planning, this paper fosters synchronized decision-making between departments providing the firm with optimal overall decisions.

The solution discussed in this paper helps to avoid making decisions that overlook the impact of the business environment. Since we model pricing and operations in competitive environments, the pricing strategies derived from our method withstand the effect of interaction with other firms. Although previous research has addressed pricing under competition, we go further by including operations planning on the competitive interactions of the firm.

This paper can also be of interest to firms launching new products in competitive markets, because the demand for new products is in general highly sensitive to the posted price. Decision makers can use joint dynamic pricing and production/inventory planning to learn about the demand. Thus, pricing, planning and actual demand from previous periods could be used to calibrate the model for the price sensitivity of the demand.

Our work opens interesting research avenues. First, a natural extension of our work is to study the capacitated version of the lot-sizing problem. In this line, it could be interesting to incorporate well-studied valid inequalities for making the computations of the LSP faster and to link them with the pricing of the firm and competitors. Second, it would be useful to examine the trade-off between price and delivery time when working in a competitive environment. In practice, customer demand depends on the price, but it can also depend on other factors, such as the promised delivery time. In that scenario, promising a shorter time will bring more demand. However, this will require additional efforts when managing operations. Another way to extend this research would be to study how to obtain an NE when the firms have incomplete information about their competitor's strategies. The procedure for obtaining such an NE has a similar structure to the problem we study. However, the complexity of reaching the equilibrium will increase due to the uncertainty. Finally, we believe is interesting to analyze pricing equilibrium under competition with uncertain demands. We perceive two options for planning operations under such uncertainty: (i) to model the operations of each firm as a Stochastic LSP (see e.g. Guan et al. (2006)) in which the demand is modeled as a probability distribution, (ii) to assume that the demand will be in a certain range of values, and model the operations of a firm as a Robust LSP (see e.g. Bertsimas and Thiele (2006)).

\section{Acknowledgements}


This research was partially supported by the ARC project "Managing Shared Resources in Supply Chains"(Project n. ARC 08-13-008). The authors would like to thank the review team and the editor for

\section{References}

Adida, E., Perakis, G., 2006. A robust optimization approach to dynamic pricing and inventory control with no backorders. Mathematical Programming 107, 97-129.

Adida, E., Perakis, G., 2010. Dynamic pricing and inventory control: uncertainty and competition. Operations Research 58, 289-302.

Bertsimas, D., Thiele, A., 2006. A robust optimization approach to inventory theory. Operations Research 54, 150-168.

Bhattacharjee, S., Ramesh, R., 2000. A multi-period profit maximizing model for retail supply chain management: an integration of demand and supply-side mechanisms. European Journal of Operational Research 122, 584-601.

Bonsall, P., Shires, J., Maule, J., Matthews, B., Beale, J., 2007. Responses to complex pricing signals: theory, evidence and implications for road pricing. Transportation Research Part A: Policy and Practice $41,672-683$.

Brahimi, N., Dauzere-Peres, S., Najid, N. M., Nordli, A., 2006. Single item lot sizing problems. European Journal of Operational Research 168, 1-16.

Cachon, G., Harker, P., 2002. Competition and outsourcing with scale economies. Management Science 48, 1314-1333.

Chan, L. M. A., Max Shen, Z. J., Simchi-Levi, D., 2004. Coordination of pricing and inventory decisions: a survey and classification. In: Handbook of Quantitative Supply Chain Analysis: Modeling in the E-Business Era. Springer, Ch. 9, pp. 336-392.

Chen, C., Johnson, K., Min, K., 1995. Analyses of sensitivity and competition of an eoq model. International Journal of Systems Science 26, 1559-1570.

Chen, C., Min, K., 1994. A multi-product eoq model with pricing consideration - T.C.E. Cheng's model revisited. Computers \& Industrial Engineering 26, 787-794.

Chen, M., Chu, M., 2003. The analysis of optimal control model in matching problem between manufacturing and marketing. European Journal of Operational Research 150, 293-303.

Cheng, T., 1990. An EOQ model with pricing consideration. Computers \& Industrial Engineering 18, $529-534$.

Deng, S., Yano, C., 2006. Joint production and pricing decisions with setup costs and capcity constraints. Management Science 52, 741-756. 
Elmaghraby, W., Keskinocak, P., 2003. Dynamic pricing in the presence of inventory considerations: research overview, current practices, and future directions. Management Science 49, 1287-1309.

Feichtinger, G., Hartl, R., 1985. Optimal pricing and production in an inventory model. European Journal of Operational Research 19, 45-56.

Fredergruen, A., Meissner, J., 2009. Competition under time-varying demands and dynamic lot sizing costs. Naval Research Logistics 56, 57-73.

Gilbert, S., 1999. Coordination of pricing and multi-period production for constant priced goods. European Journal of Operational Research 114, 330-337.

Gilbert, S., 2000. Coordination of pricing and multi-period production accross multiple constant priced goods. Management Science 46, 1602-1616.

Gonzalez-Ramirez, R., Smith, N. R., Askin, R., 2011. A heuristic approach for a multi-product capacitated lot-sizing problem with pricing. International Journal of Production Research 49, 1173-1196.

Guan, Y., Ahmed, S., Nemhauser, G. L., Miller, A. J., 2006. A branch-and-cut algorithm for the stochastic uncapacitated lot-sizing problem. Mathematical Programming 105, 55-84.

Haugen, K., Olstad, A., Pettersen, B., 2007. Solving large-scale profit maximisation capacitated lot-size problems by heuristic methods. Journal of Mathematical Modelling and Algorithms 6, 135-149.

Jung, H., Klein, C., 2006. Optimal inventory policies for profit maximizing EOQ models under various cost functions. European Journal of Operations Research 174, 689-705.

Karimi, B., Fatemi Ghomia, S., Wilson, J., 2003. The capacitated lot sizing problem: a review of models and algorithms. Operations Research 43, 264-281.

Kim, D., Lee, W., 1998. Optimal joint pricing and lot sizing with fixed and variable capacity. European Journal of Operations Research 109, 212-227.

Kunreuther, H., Schrage, L., 1973. Joint pricing and inventory decisions for constant priced items. Management Science 19, 732-738.

Lanquepin-Chesnais, G., Haugen, K., Olstad, A., 2012. Large-scale joint price-inventory decision problems, under resource limitation and a discrete price set. J Math Model Algor 11, 269-280.

Lee, W., 1993. Determining order quantity and selling price by geometric programming: optimal solution, bounds, and sensitivity. Decision Sciences 24, 76-87.

Lee, W., 1994. Optimal order quantities and prices with storage space and inventory investment limitations. Computers \& Industrial Engineering 26, 481-488.

Martel, A., Gascon, A., 1998. Dynamic lot-sizing with price changes and price-dependent holding costs. European Journal of Operations Research 111, 114-128. 
Mauri, A., 2007. Yield management and perceptions of fairness in the hotel business. International Review of Economics 54, 284-293.

Min, K., 1992. Inventory and pricing policies under competition. Operations Research Letters 12, 253-261.

Nash, J., 1950. Equilibrium points in n-person games. Proc. of the National Academy of Sciences 36, $48-49$.

Onal, M., Romeijn, H., 2011. Multi-item capacitated lot-sizing problems with setup times and pricing decisions. Naval Research Logistics 57, 172-187.

Pedroso, J., Smeers, Y., 2010. Equilibria on a game with discrete variables. In: Programs, Proofs, Processes. Computability in Europe 2010, pp. 326-335.

Pekelman, D., 1974. Simultaneous price-production decisions. Operations Research 22, 788-794.

Ray, S., Gerchak, Y., Jewkes, E., 2005. Joint pricing and inventory policies for make-to-stock products with deterministic price-sensitive demand. International Journal of Production Economics 97, 143-158.

Sandholm, T., Gilpin, A., Conitzer, V., 2005. Mixed-integer programming methods for finding nash equilibria. Computer Science Department Paper 1458.

Thomas, J., 1970. Price-production decisions with deterministic demand. Management Science 16, 747750.

Transchel, S., Minner, S., 2011. Economic lot-sizing and dynamic quantity competition. International Journal of Production Economics 133, 416-422.

Upasani, A., Uzsoy, R., 2007. Incorporating manufacturing lead times in joint production-marketing models: a review and some future directions. Ann Oper Res 161, 171-188.

Vanthienen, L. G., 1975. Simultaneous price-production decision making with production adjustment costs. TIMS XX international meeting.

Whitin, T. M., 1955. Inventory control and price theory. Management Science 2, 61-68.

Wolsey, L. A., 1995. Progress with single-item lot-sizing. European Journal of Operational research 86, $395-401$.

Yano, C. A., Gilbert, S. M., 2005. Coordinated pricing and production/procurement decisions: a review. In: Managing Business Interfaces. Springer, pp. 65-103. 